\title{
1. Including Kinetic Ion Effects in the Coupled Global Ionospheric
} Outflow Solution

\author{
A. Glocer $^{1}$, G. Toth ${ }^{2}$, and M.-C. Fok ${ }^{1}$ \\ ${ }^{1}$ NASA/GSFC, Greenbelt, Maryland, USA
${ }^{2}$ Climate and Space Sciences and Engineering, University of Michigan,Ann Arbor, MI, USA.
}

3

$6 \quad$ Key Points:

7 - We present a global ionospheric outflow model with kinetic ions and wave-particle $8 \quad$ interactions.

$9 \quad$ - The code uses a hybrid parallelization scheme to achieve fast execution.

10 - It is the first two-way coupled global kinetic outflow code coupled to an MF-MHD 11 magnetosphere.

This is the author manuscript accepted for publication and has undergone full peer review but has not been through the copyediting, typesetting, pagination and proofreading process, which may lead to differences between this version and the Version of Record. Please cite this article as doi: 10.1002/2018JA025241

Corresponding author: A. Glocer, alex.glocer-1@nasa.gov

This article is protected by copyright. All rights reserved. 


\begin{abstract}
We present a new expansion of the Polar Wind Outflow Model (PWOM) to include kinetic ions using the Particle-in-Cell (PIC) approach with Monte Carlo collisions. This implementation uses the original hydrodynamic solution at low altitudes for efficiency, and couples to the kinetic solution at higher altitudes to account for kinetic effects important for ionospheric outflow. The modeling approach also includes wave-particle interactions, suprathermal electrons, and an hybrid parallel computing approach combining shared and distributed memory paralellization. The resulting model is thus a comprehensive, global, model of ionospheric outflow that can be run efficiently on large supercomputing clusters. We demonstrate the model's capability to study a range of problems starting with the comparison of kinetic and hydrodynamic solutions along a single field line in the sunlit polar cap, and progressing to the altitude evolution of the ion conic distribution in the cusp region. The interplay between convection and the cusp on the global outflow solution is also examined. Finally, we demonstrate the impact of these new model features on the magnetosphere by presenting the first 2-way coupled ionospheric outflow-magnetosphere calculation including kinetic ion effects.
\end{abstract}

\title{
1 Introduction
}

All of the plasma in Earth's magnetosphere derives its origin from one of two sources: the solar wind or the planet itself. The escape of plasma from the ionosphere to populate the magnetosphere is often referred to as ionospheric outflow. In contrast to the solar wind plasma which is mostly comprised of $\mathrm{H}^{+}$, magnetospheric plasma of ionospheric origin can consist of $\mathrm{H}^{+}$and heavier species such as $\mathrm{O}^{+}$. The origin of near-Earth plasma is an issue of fundamental importance as it impacts every facet of the space environment system. Indeed, ionospheric plasma is found to affect the ring current [e.g., Nosé et al., 2005], wave growth in the inner magnetosphere [e.g., Kozyra et al., 1984], reconnection [e.g., Shay and Swisdak, 2004], and flows in the magnetosphere [e.g., Garcia et al., 2010], to name only a few. While the relative importance of the ionospheric and solar wind source of plasma is still hotly debated, it has been suggested that the ionosphere is a fully adequate source of plasma to fill the magnetosphere [Chappell et al., 1987]. This is particularly evident during geomagnetic storms where the large quantities of $\mathrm{O}^{+}$in the magnetosphere are indicative of the strength of the ionospheric source [Lennartsson et al., 1981]. Given the importance of ionospheric outflows, the development of coupled magnetosphere-ionosphere models that account for the mass transport and feedback between these regions is critical.

An empirical specification of ionospheric outflow is the most straightforward approach to include an ionospheric mass source in a global magnetospheric simulation. The simplest empirical specification is to just set an average density and temperature with a zero outflow velocity uniformly around the inner simulation boundary. This approach was used in the first model tracking ionospheric $\mathrm{O}^{+}$as a population in the magnetosphere [Winglee, 1998], and was studied in great detail by Welling and Liemohn [2014]. More complex empirical specifications seek to link the outflowing flux to energy inputs, for instance electron precipitation and Poynting flux [Zheng et al., 2005; Strangeway et al., 2005]. This approach is extremely popular [e.g., Fok et al., 2006; Brambles et al., 2011] as it allows for a spatially and temporally varying, causally driven, outflow specification while dispensing with the difficulty of modeling the physical processes that actually drive the outflow. While the empirically specified outflow approach has a number of advantages, it suffers from some significant drawbacks. For instance, the empirical specifications are often based on data sets that encompass a limited time period, are unable to distinguish between species type, and assume that the outflow flux changes instantaneously when the energy input changes. Despite these limitations, models using empirically specified fluxes have demonstrated that including outflow in a magnetosphere simulation can have major impacts. 
It is also possible to use first-principles based modeling in order to specify the inner boundary conditions to reflect the outflowing plasma from the ionosphere. Glocer et al. [2009a] was the first to implement this approach, coupling a hydrodynamic polar wind model with the BATS-R-US global magnetosphere model. This coupled model was later used in a number of subsequent studies [e.g., Welling et al., 2011; Ilie et al., 2013]. Recently, Varney et al. [2016] coupled an eight-moment outflow model into the LFM global magnetosphere model enabling transversely accelerated ions to be included in the calculation. The advantage of the first-principles based modeling outflow specification is that the plasma can be tracked from the origin in the ionosphere to its fate in the magnetosphere, and the underlying physical processes can be examined. The primary disadvantage of this approach is the higher computational expense.

The two models referred to above to calculate the ionospheric outflow solution are both hydrodynamic in nature. However, one of the longest running controversies in polar wind modeling is the use of and applicability of hydrodynamic models for describing polar outflows [Donahue, 1971; Lemaire and Scherer, 1973]. Shortly after the introduction of the supersonic polar wind by Axford [1968], Banks and Holzer [1968] introduced a hydrodynamic model to support this conjecture. However, the use of the hydrodynamic approach was objected to by others including Dessler and Cloutier [1969]. In essence, they argued that if the hydrodynamic solution does not correctly describe the geocorona, it should not be able to described the ionized outflow. Moreover, the hydrodynamic solution should only be valid when there exist sufficient collisions such that the ion distribution function can be described as a perturbation around a Maxwellian distribution function. Despite its seeming inapplicability, however, early attempts to compare the polar wind $\mathrm{H}^{+}$ predictions from hydrodynamic models and kinetic models showed the two approaches yielded surprisingly similar results [Marubashi, 1970; Holzer et al., 1971]. Nevertheless, non-Maxwellian distributions such as ion conics, beams, and double hump distributions are known to exist in the outflowing plasma and should be included when using a physicsbased model to specify the outflow [e.g., Barakat et al., 1995]. So far only Welling et al. [2016], has made any attempt to use a model with a kinetic ion description to represent the outflow for a global magnetosphere calculation. They use the Generalized Polar Wind (GPW) model of Barakat and Schunk [2006] which has a fluid description below 1200km, and a kinetic particle in cell (PIC) description above. This approach is thus capable of including important ion kinetic effects into the coupled outflowing plasma solution, but the coupling of GPW and the BATS-R-US magnetosphere is strictly one-way. In other words, GPW is independently executed and the results are read from files to set the MHD inner boundary condition. As a result, this work does not include the feedback of the magnetosphere to the outflow calculation. To date, there does not exist an outflow model with a kinetic ion description that is fully integrated with the magnetosphere calculation.

This paper has two main objectives. First, to introduce the expansion of the Polar Wind Outflow Model (PWOM) [Glocer et al., 2007, 2009a, 2012] to include kinetic ions using the PIC approach with Monte Carlo collisions. The model also includes the effect of wave-particle interactions, suprathermal electrons, and a hybrid parallelization scheme that combines shared and distributed memory parallel computing to achieve fast execution. Second, we demonstrate that the newly expanded PWOM code is two-way coupled with the multi-fluid BATS-R-US magnetosphere model enabling new studies of the role of kinetic ion effects in the global outflowing plasma solution, the subsequent impact on the magnetosphere, and the eventual feedback on the outflow calculation.

\section{Adding Kinetic Ions to the Polar Wind Outflow Model}

The previous version of PWOM, described by Glocer et al. [2012] and prior publications, is restricted to the gyrotropic transport equations for the ions. Such a hydrodynamic approach can easily include chemistry and collisions at minimal computational expense, but is only strictly valid where collisions are important. The extent of this region is de- 


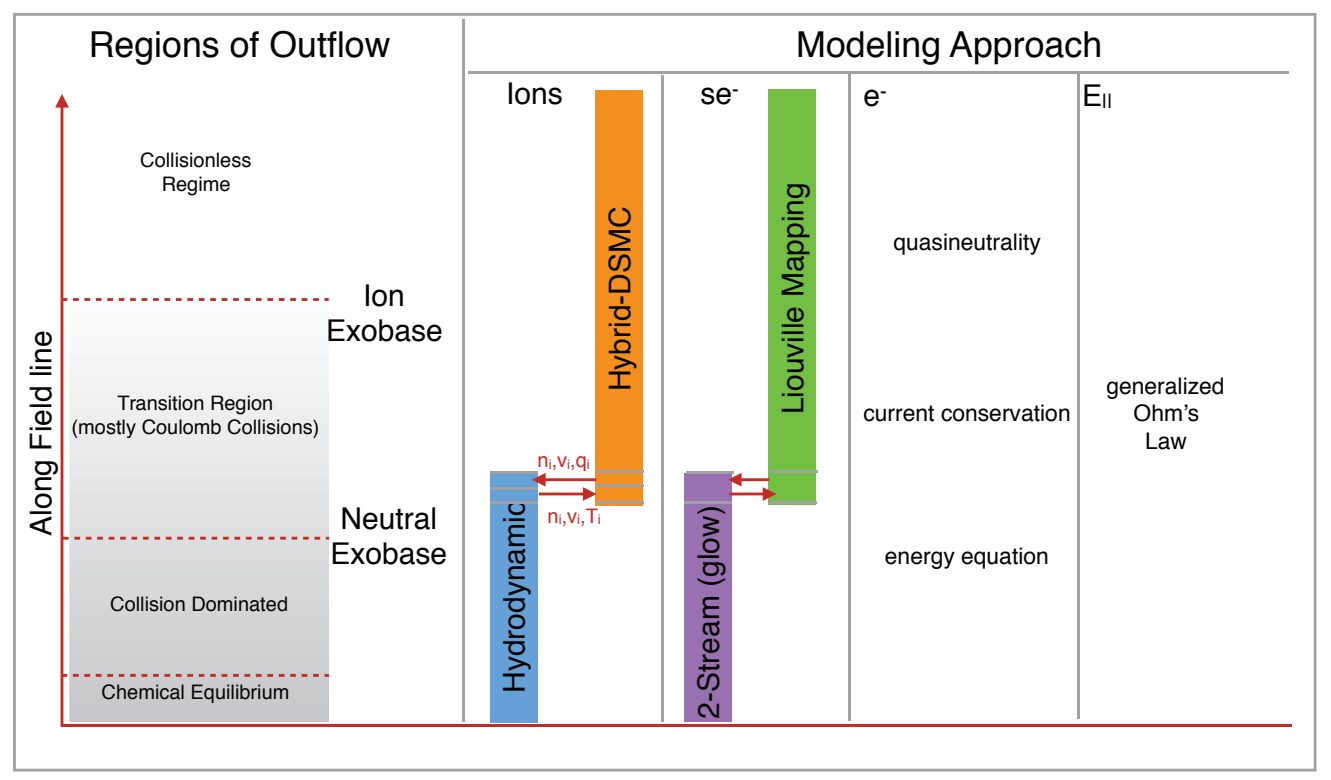

Figure 1. A schematic depiction of the the different outflow regions along field line (left) together with the modeling approach (right) for ions, suprathermal electrons $\left(\mathrm{se}^{-}\right)$, thermal electrons $\left(\mathrm{e}^{-}\right)$, and the ambipolar electric field( $\mathrm{E}_{\|}$.

fined by the concept of the ion exobase or baropause, located at about $2000 \mathrm{~km}$, described by Lemaire and Scherer [1970] and others as the altitude at which the mean free path of ions is equal to the scale height. Below this height the hydrodynamic approach is perfectly valid while above it the validly becomes increasingly suspect. Moreover, the hydrodynamic solution is not capable of modeling non-Maxwellian distributions such ion conics frequently observed above the cusp and auroral regions. We therefore expand the PWOM model to use the hydrodynamic approach only at low altitudes where it is both valid and efficient, and transition to a kinetic PIC solution at higher altitudes. This approach is conceptually similar to that described by some other models [e.g., Estep et al., 1999; Barakat and Barghouthi, 1994] but has a number of advantageous features. This section describes the modeling approach in detail.

Figure 1 provides a schematic illustration of modeling approach along a single field line. On the left of Figure 1, an illustration of the different regions of outflow is provided for context. Below 1000km PWOM uses its original hydrodynamic approach to solve the gyrotropic transport equations for ions [Gombosi and Nagy, 1989]. Above 1000km, PWOM uses a Direct Simulation Monte Carlo (DSMC) approach which obtains a kinetic solution by following guiding center macro-particles for each ion species. As the electric field is calculated self-consistently, as in a hybrid-PIC simulation, we adopt the nomenclature 'hybrid-DSMC' method as a short hand although some publications also refer to this approach as 'Mac-PIC'. The hydrodynamic model provides the lower boundary condition for the hybrid-DSMC calculation, and the hybrid-DSMC model provides the upper boundary condition for the hydrodynamic model. Each macro particle is advanced under the influence of gravity, the force associated with the parallel electric field, and the mirror force according to the standard equation of motion for a gyro-averaged particle

$$
m_{i} \frac{\partial v_{i \|}}{\partial t}-q_{i} E_{\|}+\frac{G m_{i} M_{\text {planet }}}{r^{2}}+\mu_{i} \frac{\partial B}{\partial s}=0
$$

where $i$ is the ion species index, $m_{i}$ is the ion mass, $v_{i}$ is the ion velocity, $t$ is time, $q_{i}$ is the ion charge, $E_{\|}$is the parallel electric field, $G$ is the gravitational constant, $M_{\text {planet }}$ is 


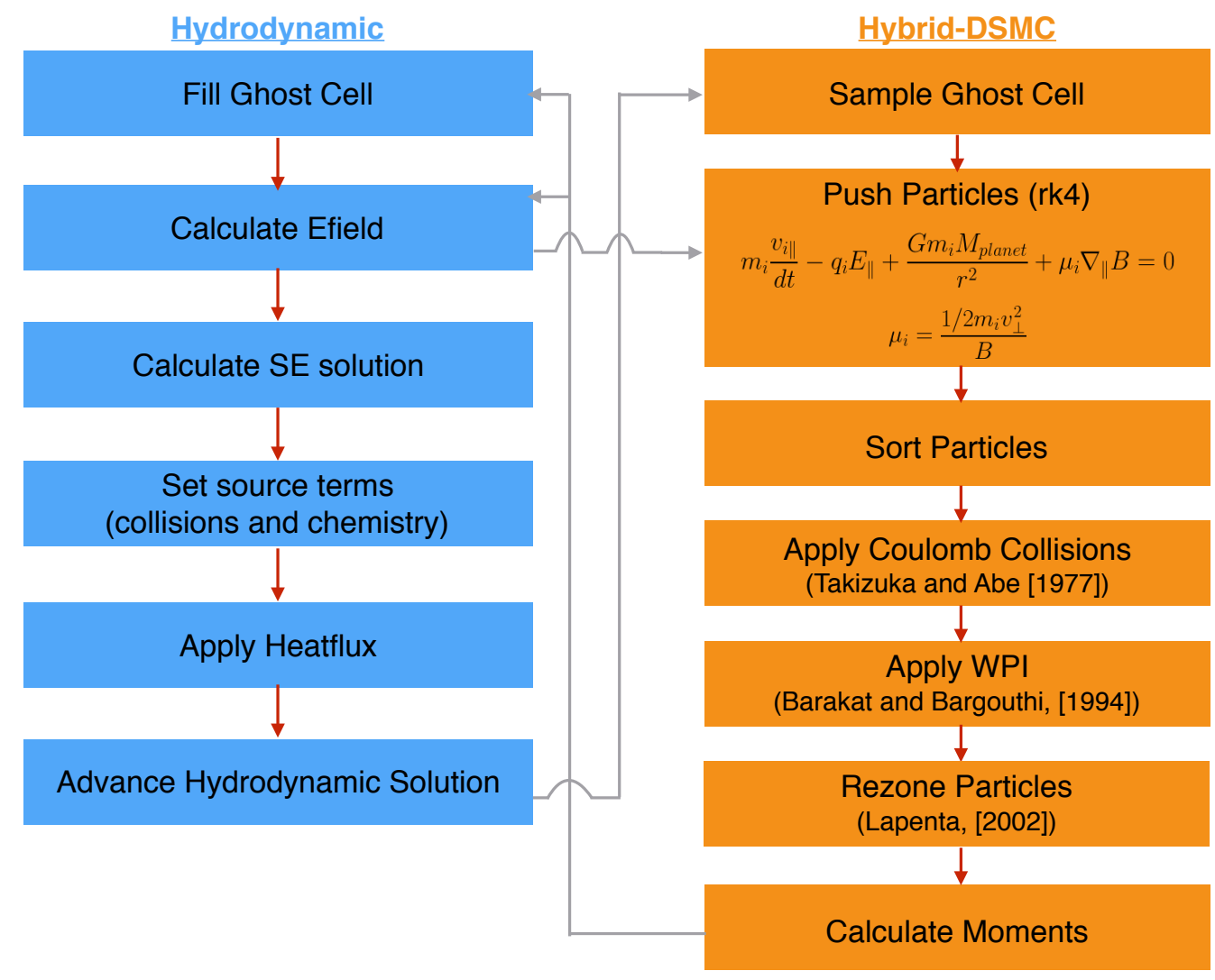

Figure 2. Block diagram of the calculation flow. The original PWOM hydrodynamic portion of the calculation is on the left (blue), and the new hybrid-DSMC portion of the calculation on the right (orange). The connection between these two approaches are shown with gray arrows. Focusing on the new hybrid-DSMC portion of the calculation, references for specific algorithms adapted into the present calculation are provided.

the mass of the planet, $r$ is the radial distance measured from the center of the planet, $B$ is the magnetic field magnitude, and $s$ is the distance along the magnetic field. $\mu_{i}$ is the particle's first adiabatic invariant defined by

$$
\mu_{i}=\frac{m_{i} v_{\perp}^{2}}{2 B}
$$

The inclusion of Coulomb collisions and wave particle interactions are provided as a separate operation from the particle push using the Monte Carlo approach.

Figure 2 summarizes the flow of the calculation using a block diagram. The left side shows the steps of the hydrodynamic portion of the model in blue, while the right side shows the steps of the hybrid-DSMC portion of the calculation in orange. As the hydrodynamic portion has been presented in our previous work, we focus on the hybrid-DSMC part of the code. The first step in this portion is to randomly sample macro-particles for each ion species in the lower ghost cell (sometimes referred to as a boundary condition cell) from a Maxwellian distribution whose density, velocity, and temperature are determined from the hydrodynamic model. Particle weights for the sampling are determined by 
dividing the number of true particles in the cell by the target number of macro-particle per cell. Every time the ghost cell is sampled any previously existing macro-particles in the ghost cell are discarded and replaced with the newly sampled macro-particles. We then 'push' our macro-particles according to the guiding center equation of motion given above using a 4th order Runge-Kutta (RK4) scheme. Particles are then sorted into cells so that collisions and wave-particle interactions can be applied. These processes are described in more detail in the following subsections. To maintain good statistics with reasonable computational expense, we use the macro-particle 'rezoning' technique described by Lapenta [2002] to either split or join particles until we are within a predefined tolerance of our target number of macro-particles per cell; splitting a macro-particles means converting it into two new macro-particles each representing half of the original number of true particles, while joining macro-particles means combining two macro-particles into a single macro-particle representing the same number of true particle. Finally, we calculate moments (density, velocity, temperature, and heat flux) for each ion species from the macroparticles to couple with the hydrodynamic code.

As noted by Lapenta [2002], joining macro particles is delicate operation and thus requires some further elaboration. The main idea is to join macro particles in a way that leaves the new set of particles equivalent. In other words, the new set of macro particles after the joining operation should ideally have the same velocity space distribution and moments as the old set. We therefore only select particles to join that are close to each other in velocity space. This is achieved by first sorting particles in a given altitude cell into velocity space bins whose size is much smaller than the thermal speed. The particle with the lowest statistical weight $\left(\mathrm{w}_{1}\right)$ in the altitude cell is then selected to be combined with the next lowest weight particle $\left(\mathrm{w}_{2}\right)$ in the same altitude cell and velocity space bin. In this way we systematically eliminate particles with low statistical weight by joining them to particles with higher statistical weight. The new particle has a combined statistical weight $\left(\mathrm{w}_{c}\right)$ of

$$
w_{c}=w_{1}+w_{2}
$$

and a new position and velocity given by the statistically weighted average of the old particle's values. This approach conserves number density and momentum exactly, but not energy. However, by only combining particles that are close together in velocity space, any error in the energy is in practice quite small. Indeed, this algorithm is largely equivalent to one described by Lapenta [2002] (called algorithm C1 in that paper), and was found in their tests to do an excellent job in preserving both the moments and the shape of the velocity space distribution. Our own simple testing of the algorithm confirms this. In our test, we initialize the particles in a given cell according to a drifting Maxwellian distribution with a specified density, velocity, and temperature. The particles in that cell are then split 60 times followed by being joined 60 times. Each operation works on 5\% of the available particles, which is typical in a PWOM simulation. The error in the density, velocity, and temperature after each split or join operation is tracked. The split operations exactly conserve mass, momentum and energy and thus preserve all three moments to round off error as expected. The join operations exactly conserve the mass and momentum and therefore preserve the density and velocity moments to round off error. The cumulative error in the temperature after 60 join operations was found to be approximately $0.05 \%$. As the particle rezoning operation is only applied at most once a minute, to reduce computational overhead, and not every rezoning operation involves joining particles, these small errors can be regarded as a conservative estimate of the maximum error introduced during an hour of simulation. Finally, we note that visual inspection comparing the final and original particle velocity space distributions also demonstrates at most miniscule change.

One critical aspect of the calculation flow is the interface between the hydrodynamic model and kinetic model. As noted in the description above, the top computational cell of the hydrodynamic model provides the moments to define a distribution function from which macro-particles are sampled to fill the lower ghost cell of the kinetic model. Like- 
wise, the upper ghost cell of hydrodynamic model is filled by moments calculated at the bottom of the computational domain of the kinetic model. However, only the density and velocity moments from the kinetic model are used directly, but not the temperature. Instead, the temperature is set such that the heat flux in the hydrodynamic model matches the heat flux in the kinetic model at the interface, specifically

$$
-\kappa_{i} \nabla T_{i}=q_{i}^{\text {kinetic }}
$$

where $\kappa_{i}$ is the heat flux coefficient for species $i$. This approach, described first by Estep et al. [1999], is critical for two-way coupling as it ensures that mass and energy flux are conserved across the interface. If instead the temperature or pressure from the kinetic model was used to set the upper boundary on the hydrodynamic calculation, the Fourier heat flux would not match the kinetic heat flux, resulting in a non-zero divergence of the heat flux at the interface, and subsequent build up of energy. By selecting the temperature in the upper ghost cell of the hydrodynamic model such that the heat flux is conserved across the interface the divergence of the heat flux is zero, the energy flux is conserved across the interface, and no build up of heat occurs.

While the block diagram adequately describes the calculation flow, some portions of the calculation require further description. Therefore the following subsections detail the treatment of the electrons, the application of Coulomb collisions, and the inclusion of wave-particle interactions.

\subsection{Treating the Electrons}

The electrons are split into two populations: thermal electrons and suprathermal electrons (SEs). Together with the ions, these two electron populations must satisfy quasineutrality and current conservation. Additionally we solve an energy equation for the electron temperature, and the electric field is represented using a generalized Ohm's law derived from the steady state momentum equation. As this approach was recently described by Glocer et al. [2012] and Glocer et al. [2017] we will not repeat the complete description here, but instead focus our discussion on the treatment of the SEs which is somewhat different than in our previous efforts.

The idea that suprathermal electrons (electrons with energies much greater than thermal energies) can cause heavy ion outflows was suggested by Axford [1968]. The SEs come from three sources including photoionization of the atmosphere, precipitating electrons of magnetospheric origin (polar rain, cusp, and auroral electrons), and secondary electrons produced by impact ionization of the neutral atmosphere. They affect the outflowing solution through the ambipolar electric field and energy deposition from the suprathermal population to the thermal population. Even very small concentrations of these electrons can have a dramatic impact on the outflow [Khazanov et al., 1997]. The SE population, however, requires a special treatment. In our past work this population is either specified at the base of the field line from an external calculation and then mapped to higher altitudes, as was done in Glocer et al. [2012], or a self consistent kinetic equation is solved, as was done in Glocer et al. [2017]. The former approach does not self consistently treat the ionization and suprathermal electron production, but is very fast. The latter approach is much more physical, but can be quite onerous computationally. We therefore adopt a compromise approach to the calculation of the SEs and embed a two-stream treatment of this population.

The two-stream approach to the SE population was first presented by Banks and Nagy [1970] and Nagy and Banks [1970]. In this technique the SEs are split into an upgoing stream and a down-going stream assumed to be represented by an average, usually isotropic, pitch-angle. The two-streams include the photo-production, secondary production, collisions, and transport at each altitude. They are moreover coupled by a backscatter coefficient which represents the transfer of electrons from the up-going stream to the 
down-going stream through collisions. There are a number of two-stream representations in the community, but the specific implementation embedded into PWOM is the GLOW model [Solomon et al., 1988; Solomon, 2017].

The inclusion of the GLOW model into PWOM is virtually identical to the inclusion of the fully kinetic SE solution described by Glocer et al. [2017]. In both approaches the electric field as well as the thermal electron density and temperature profiles are passed to the SE model. If a precipitation is present, it is specified as an upper boundary condition on the SE model. The SE model then finds the SE solution and passes back to PWOM the ionization rate (which matches the SE production rate), the SE number density, number flux, as well as the energy deposition and momentum transfer to the thermal electron population. To use GLOW in this approach only required a few small changes. First, we had to put the code into a 'wrapper' so that each PWOM field line can call an independent GLOW solution. Second, GLOW had to be modified to use PWOM provided thermal electron parameters rather than the parameters from the IRI empirical model [Bilitza et al., 1990]. Third, above $1000 \mathrm{~km}$ we use Liouville mapping to extend the GLOW solution to higher altitudes where the changing magnetic field strength and electric potential drop make the isotropic two-stream representation less valid. Finally, we take the reflected portion of the SE solution from the Liouville mapping and impose that as an upper boundary condition on the GLOW calculation. The calculation is then repeated and the boundary condition is updated again. This iteration continues until the change from one solution to the next is smaller than a specified tolerance.

Including the two-stream electron representation is a compromise between computational speed and completeness. The two-stream representation allows for a self-consistent representation of the ion and SE production, SE transport, as well as the collisional interactions. This makes it much better than relying on an externally specified SE solution. However, the two-stream approach is not as physically complete as the fully kinetic representation. In compensation, two-stream approach is orders of magnitude faster while providing a fully adequate description of the SEs. This speed up is critical as the addition of the kinetic ion treatment to PWOM is itself quite computationally demanding and every bit of code speedup is essential in order to achieve our goal of a fast global solution with ion kinetic effects.

\subsection{Including Coulomb Collisions}

The effect of Coulomb collisions on the guiding center macro-particle ion velocities is included probabilisticly using the Monte Carlo approach. This point is particularly important as the hand-off between the hydrodynamic and DSMC approach, where the upper and lower boundary cells respectively are filled by the other model, must occur in a region where both approaches are valid. Including collisions in our kinetic ion description enables both it and the hydrodynamic model to be valid in the transition region making that a suitable location for transition between physical approaches. Only Coulomb collisions are included by the model currently. Neutral collisions could be included as well, but as we choose the transition altitude to be situated above the neutral exobase but below the ion exobase, including only Coulomb collisions is sufficient.

The specific algorithm for applying the Coulomb collisions is based on the widely used technique of Takizuka and Abe [1977]. In this approach all ions in a given cell are randomly paired and collided in a given timestep. The scattering angle, $\Theta$, of a given collision is provided by $\delta=\tan (\Theta / 2)$ where $\delta$ is a uniformly distributed random variable with a mean of zero and variance given by

$$
\left\langle\delta^{2}\right\rangle=\frac{q_{i}^{2} q_{j}^{2} n_{L} \lambda}{8 \pi \epsilon_{0}^{2} m^{2} u^{3}} \Delta t
$$


where the subscript ' $i$ ' or ' $j$ ' represents the ion index, $q_{i}$ is the charge, $n_{L}$ is the minimum of ion densities $i$ and $j$ in the case they are of different species, $\lambda$ is the Coulomb logarithm, $m_{i j}$ is the reduced mass, $u$ is the relative velocity of ion $i$ and $j$, and $\Delta t$ is the timestep. An interested reader can find full details and discussion of special cases in Takizuka and Abe [1977].

In our code we allow macro-particles to have arbitrary weights to accomodate the particle rezoning and must therefore consider an expansion of the algorithm of Takizuka and Abe [1977] which assumes identically weighted particles. Miller and Combi [1994] first expanded that algorithm for the case of particles with two weights using a rejection technique. Rejection methods are commonly used in Monte Carlo models with differently weighted particles to avoid over counting collisions. For example, if a macro-particle representing 10 real particles collides with a macro-particle representing 100 real particles then the resulting collision would not conserve energy and momentum since the 'heavier' macro-particle weighs more strongly in the moment calculations. Therefore some collisions must be probabilistically rejected. For variably weighted macro-particle, where each macro-particle has a different weight, we use the rejection probabilities calculated by Nanbu and Yonemura [1998]. As a result, when working with equally weighted macroparticles we conserve energy and momentum exactly with each collision, while when working with variably weighted particles we conserve energy and momentum on the average.

To verify that the Coulomb collisions are calculated properly in our model, we apply our implementation to a standard test problem: The equilibration of temperature. In this problem, we consider two populations ('i' and 'e') with equal number densities, but different masses and temperatures and see how long it takes for the temperatures to come to equilibrium. Transport is disabled for this test problem. We consider two cases, one with equally weighted macro-particles and another with each species split into two differently weighted populations yielding 4 total weights. The parameters for the test are chosen to be consistent with those used by Miller and Combi [1994] and Takizuka and Abe [1977] and are given by $T_{i}=2 T_{e}=400 \mathrm{eV}, n_{i}=n_{e}=0.5 \times 10^{6} \mathrm{~cm}^{-3}, m_{i}=4 m_{e}$.

Figure 3 presents the results of the test showing the temperature difference, normalized to the starting temperature difference, over time, normalized by the collision frequency $\left(v_{0}\right)$. The normalized temperature difference starts at 1 and continually reduces until the two species come to equilibrium. Note that the solution is nearly identical regardless of whether all macro-particles have the same weight or if various weights are used for macro-particles. Also plotted is the result of Miller and Combi [1994] and the analytic solution to this problem, assuming each population is represented by a Maxwellian, given by

$$
\frac{T_{i}-T_{e}}{T_{i 0}-T_{e 0}}=\exp -2.0 v_{e q} t
$$

where $v_{e q}=\left(8 / 3 \pi^{1 / 2}\right)\left(m_{e} / m_{i}\right)\left[1+m_{e} / m_{i}\left(T_{i} / T_{e}\right)\right]^{-3 / 2} v_{0}$ is the temperature equilibration frequency. Our calculation is in excellent agreement with the analytic solution during the early phase of the equilibration and with the prior solution of Miller and Combi [1994] at all times. As explained by Miller and Combi [1994], after the initial phase of the equilibration, the two populations are no longer adequately described as Maxwellian and therefore the deviation from the analytic solution is expected. Our excellent agreement with the previously published result, however, gives us confidence that our implementation of the Coulomb collisions is correct. We also note that we were able to obtain equally good agreement for other parameter choices, but the case already presented is sufficient to provide verification. 


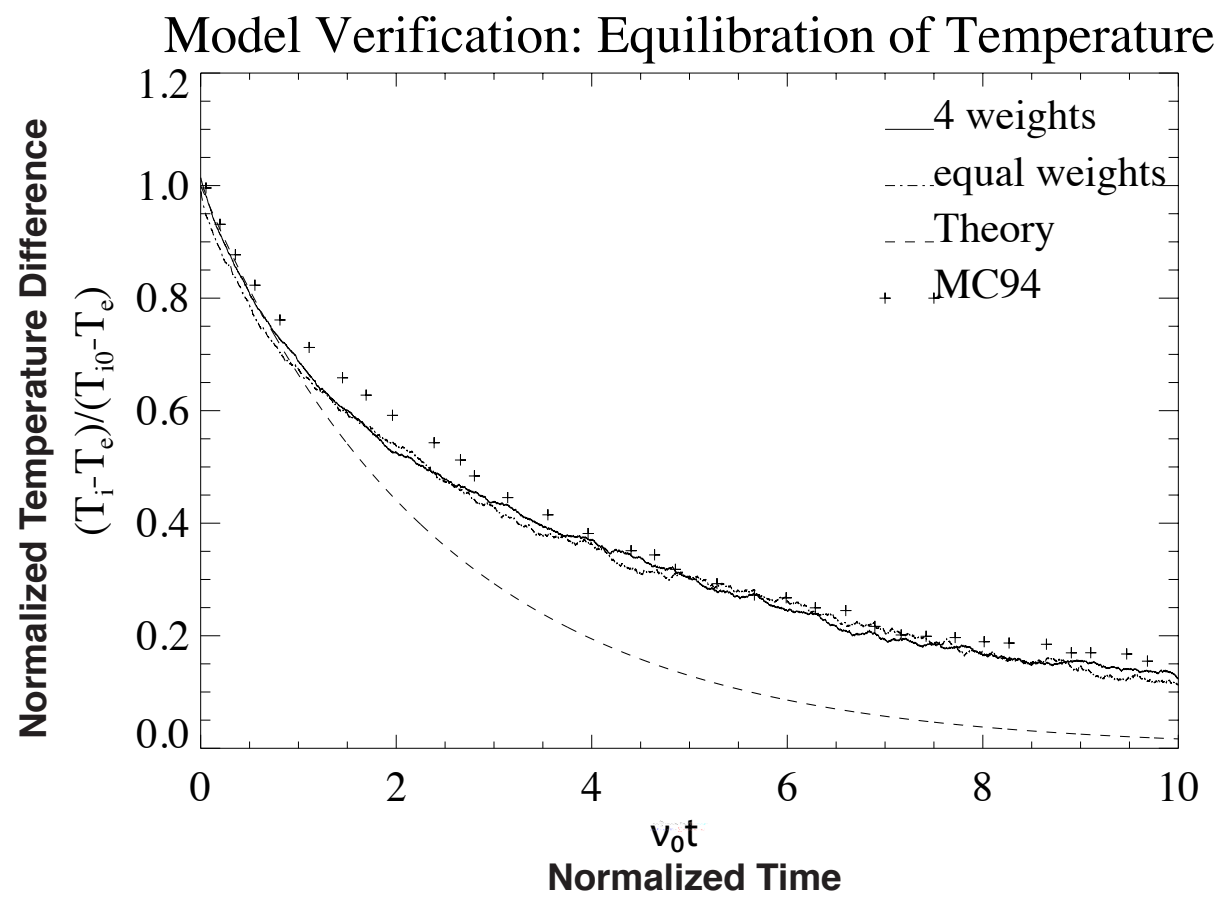

Figure 3. Verification of the implementation of the collision operator using the equilibration of temperature test. Cases with equally weighted particles (solid) and with four different weights (dash-dot) are compared with analytical theory (dashed) and with previously published results of Miller and Combi [1994] (+).

\subsection{Inclusion of wave-particle interactions}

Resonant wave-particle interactions (WPI) are widely considered to be a major pathway of ion heating and acceleration in the cusp and auroral region. The energy for this interaction is derived from broadband wave turbulence observed along field lines with power concentrated below the ion cyclotron frequency [Gurnett et al., 1984]. Ions resonating with a portion of the wave spectrum can be heated perpendicularly and then accelerated by the mirror force to form conics [Retterer et al., 1987]. While WPI of this type is impossible to add to the previously used hydrodynamic approach owing to the assumption of a scalar pressure and hence no perpendicular heating or mirror force, it is very natural to add to the DSMC model outlined above. We implemented the approach described by Retterer et al. [1987], Crew et al. [1990], and Barakat and Barghouthi [1994] who include the wave heating by randomly perturbing the perpendicular velocity such that variance of the perturbation is given by $\left\langle\left(\Delta v_{\perp}\right)^{2}\right\rangle=4 D_{\perp} \Delta t$, where $v_{\perp}$ is the perpendicular velocity, $D_{\perp}$ is the quasi-linear diffusion coefficient associated with the power spectral density (see Retterer et al. [1987]), and $\Delta t$ is the time step of the calculation. We note that the values of $D_{\perp}$ provided by Barakat and Barghouthi [1994], are based on a literature search for typical values of the wave power spectral density in different regions and is therefore a reasonable choice on the average. However, there is significant uncertainty in the exact altitude profile for the wave power, and the temporal variation is not accounted for. Indeed a major shortcoming of current models is that the wave heating parameter is not tied in any way to the magnetospheric input [Barakat and Barghouthi, 1994; Varney et al., 2016]. Nevertheless, this implementation is sufficient for the present work, and the choice of diffusion coefficients can easily be updated as new forms are developed. 


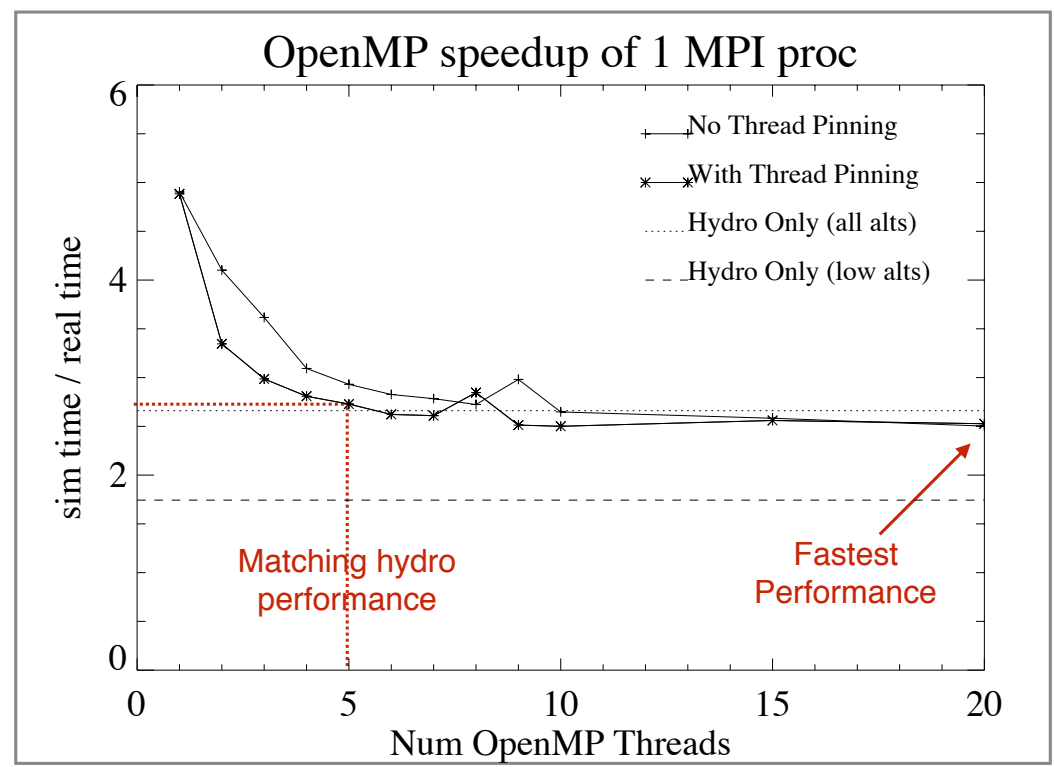

Figure 4. OpenMP speedup of a single MPI process showing the ratio of simulated to real time vs the number of OpenMP threads on an ivy bridge node on the NAS Pleiades supercomputer. Results are shown with and without careful thread pinning. For comparison, the horizontal lines show the performance of the original hydrodynamic PWOM (with no coupled particles). Note that with only 5 OpenMP threads, the coupled fluid-kinetic model is able to match the performance of the hydrodynamic model alone.

\subsection{Multiple layers of parallelization for fast execution}

A common concern when using kinetic representations in global scale simulations is execution time. In other words, the simulation must be able to run sufficiently fast on available computational resources such that all the simulations in the work plan can be accomplished. Our newly expanded PWOM code with suprathermal electrons and kinetic ions takes advantage of a hybrid of shared and distributed memory parallelization to accomplish this.

We use distributed memory parallelization to separate the number of field lines in the computation among the available processes using Message Passing Interface (MPI). This level of parallelization for PWOM was first described by Glocer et al. [2009b]. As there is no need to communicate between field lines this parallelization exhibits close to ideal scaling; it is just as fast to simulate a single field line on one process as it is to simulate a thousand field lines on a thousand processes. This simplifies our objective immensely to only needing to optimize and speedup the single field line calculation. As the hydrodynamic solution is already very fast, only acceleration of the DSMC particle solution is required.

The speed up of the DSMC solution for macro-particles on a given field line is readily accomplished with shared memory parallelization using OpenMP. We primarily parallelize the macro-particle pushing, collision, and sorting operations. As each macro-particle advances within a timestep independently of any other macro-particle, and each collision pair collides independently of any other pair, these operations are very straightforward to parallelize. The sorting operation is also sped up with OpenMP.

This hybrid of shared and distributed memory parallelization enables significant speed up. As an example, Figure 4, presents the OpenMP speedup of a single MPI process executing a single field-line simulation with approximately four million particles for 
60s of simulated time. This particular scaling was run on a single ivy bridge node on NASA's Pleiades supercomputer. The y-axis shows the ratio of simulation time to real time, and the $\mathrm{x}$-axis shows the number of OpenMP threads. The dotted line shows the performance when only the hydrodynamic model is used at all altitudes. As there is no OpenMP parallelization of the hydrodynamic model, the dotted line is flat even when the number of threads increases. The fastest model performance is about 2.5 times slower than real-time for a combined fluid-kinetic solution and requires 20 OpenMP threads. However, the benefit of using more OpenMP threads decreases as more and more are used. We find that 5 OpenMP threads per MPI process represents an acceptable combination of speed and resources. This choice enables simulations 2.7 times slower than real time with 4 MPI process, and hence 4 field lines, able to fit on a single ivy bridge node; note 20 cores are on a single ivy bridge node. OpenMP parallelization of the hydrodynamic portion of the code or the use of a larger timestep, can further acclerate performance of the calculation. While simulations 2.7 times slower than real time may not sound impressive, recall that this performance is true regardless of the number of field lines, and therefore global kinetic polar wind simulations are possible at reasonable computational expense.

There are a few other notes about the computational performance of the model that require further discussion. First, at 5 OpenMP threads per field line, the combined fluidkinetic model is actually slightly faster than the hydrodynamic only model. However, this is with approximately 4.5 million particles per field line, and more OpenMp threads will be required to achieve the same performance as the number of particles is increased. Nevertheless, we believe this is a reasonable number of particles as our convergence testing (not shown) finds that the physical solution is little changed as the number of particles is either doubled or quadrupled. This number of particles is thus a reasonable test size for measuring model performance. Second, our test considers the performance with and without careful use of 'thread pinning', the binding of an OpenMp thread to specific cores to improve memory accesses. Good use of pinning can significantly improve performance as shown in Figure 4. When PWOM is run in standalone mode, thread pinning is no problem, but when run coupled to other models through the SWMF where models running on different nodes can have different numbers of processes and threads, careful thread pinning can become onerous. In the latter case it is easier to pay an efficiency penalty. Fortunately, at 5 threads per line, the performance penalty is only about $10 \%$.

\section{Results}

Our expanded modeling approach, described in the previous section, provides a first principles treatment of most major outflow mechanisms in PWOM. In the following subsections we make use of this new capability in single field line and global outflow simulations. We begin with a single field line solution in Section 3.1 and compare the hydrodynamic solution and kinetic solution of polar wind outflow under sunlit conditions. This solution is then expanded in Section 3.2 to consider the sunlit field line in the cusp and the altitude evolution of the ion conic. Section 3.3 builds on this result by looking at the global outflow solution including cusp, but not auroral, outflow driven by wave-particle interactions and soft electron precipitation. Finally, Section 3.4 presents the first results of two-way coupling the global kinetic outflow solution from PWOM to the multi-fluid BATS-R-US magnetosphere model.

\subsection{Single Line Solution - Comparing Hydrodynamic and Kinetic Solutions}

As PWOM can be run using either hydrodynamic or kinetic descriptions above 1000 $\mathrm{km}$, the comparison of these two descriptions is an interesting first test of the newly expanded model. We therefore consider a single stationary field line exposed to sunlit conditions in the polar region, invariant latitude of approximately $70^{\circ}$. Steady state solutions 


\section{Comparing Kinetic and Hydrodynamic Solution}
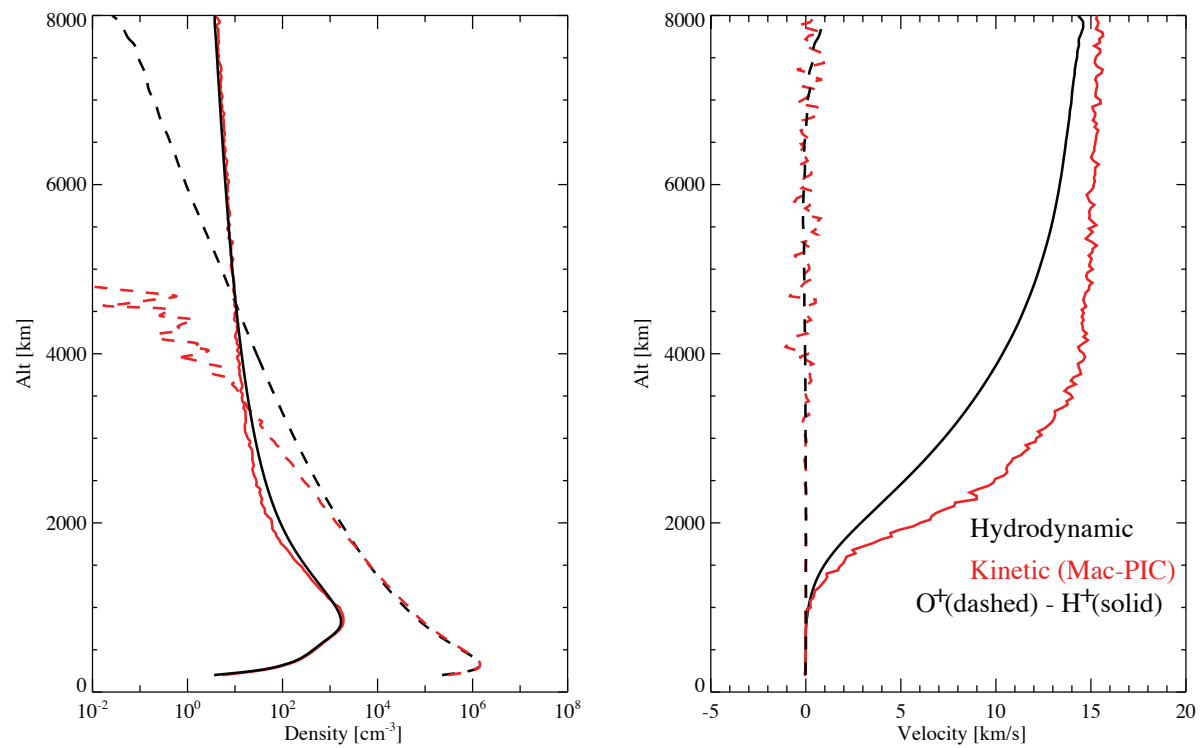

Figure 5. Altitude profiles of density and velocity for a typical sunlit polar field line comparing the hydrodynamic only solution (black) and the kinetic (above $1000 \mathrm{~km}$ ) solution (red). Both $\mathrm{H}^{+}$(solid) and $\mathrm{O}^{+}$ (dashed are shown).

of the fully hydrodynamic and the combined hydrodynamic (below $1000 \mathrm{~km}$ ) and kinetic (above $1000 \mathrm{~km}$ ) are obtained by running these time-dependent calculations until no more temporal change is visible.

Figure 5 presents the comparison of the hydrodynamic and kinetic (above 1000km) polar wind solutions. The left plot shows the altitude profile of the $\mathrm{H}^{+}$and $\mathrm{O}^{+}$densities, while the right plot shows the altitude profile of the $\mathrm{H}^{+}$and $\mathrm{O}^{+}$velocities. The hydrodynamic solution is shown in black while the kinetic solution is shown in red. The $\mathrm{H}^{+}$density solution is remarkably similar at all altitudes although some difference in the altitude evolution of the velocity is visible. In contrast the $\mathrm{O}^{+}$solution is similar in the two cases below $2000 \mathrm{~km}$, but starts to diverge above.

This result indicates that the hydrodynamic solution gives a reasonable prediction for the $\mathrm{H}^{+}$, but overestimates the $\mathrm{O}^{+}$density at high altitudes. As the $\mathrm{H}^{+}$is more easily accelerated and reaches the sonic transition in both cases, it is unsurprising that the result is similar. Indeed this is consistent with the study of Marubashi [1970] who found that the $\mathrm{H}^{+}$polar wind solution is similar in both the hydrodynamic and kinetic cases, even though the collisions are overestimated in the hydrodynamic cases. The $\mathrm{O}^{+}$, in contrast, is much more sensitive to the modeling approach. This is likely because only a small portion of the distribution function is above the escape velocity, and so the shape of the distribution much more sensitively constrains $\mathrm{O}^{+}$access to a given altitude.

\subsection{Single Line Solution - Cusp}

The cusp region is one of the most active spatial regions with regard to ionospheric outflow. One widely used study by Strangeway et al. [2005] examined multiple cusp crossings by the FAST satellite during a geomagnetic storm and found that the outflow flux correlates well with soft electron precipitation and Poynting flux. Indeed, as discussed ear- 
lier, resonant wave-particle interactions are expected above the cusp region and may lead to the formation of ion conic structures. Our model improvements are capable of handling both soft electron precipitation as well as the effects of wave-particle interactions, making this a nice model demonstration.

The physical scenario under consideration is a high latitude field line initially exposed only to sunlight that suddenly enters the cusp region. The initial condition is found by simulating a stationary, illuminated, field line for several hours until a steady solution is obtained. At time zero, soft electron precipitation, in the form of a monoenergetic beam with a mean energy of $100 \mathrm{eV}$ and energy flux of $1 \mathrm{erg} / \mathrm{cm}^{2} / \mathrm{s}$, is imposed at the top of the model. Simultaneously, the wave-particle interactions are switched on using the diffusion coefficients described above of Barakat and Barghouthi [1994]. The model is then run for 25 minutes in this configuration.

Figure 6 shows results of our combined fluid-kinetic version of PWOM for a sunlit field line that has entered the cusp and encountering soft electron precipitation and wave heating for approximately 25 minutes. As expected, densities and velocities (left part of figure) all increased compared to Figure 5 which only includes the effect of sunlight producing ionization and photoelectrons. The most significant qualitative change is seen in the $\mathrm{O}^{+}$solution which becomes greater than or equal to the $\mathrm{H}^{+}$density at all altitudes. Moreover, the velocity, negligible in the previous case, now exceeds $10 \mathrm{~km} / \mathrm{s}$ at the top of the model.

One feature of the model is that we can examine the altitude evolution of the ion distribution function in this case. As an example, the right portion of Figure 6 presents the $\mathrm{O}^{+}$velocity space distribution function at four altitudes at the end of the simulation. At $1000 \mathrm{~km}$, the location of the fluid-kinetic transition, the distribution has a typical Maxwellian shape; this is expected as a Maxwellian is fed in from the fluid portion of the code. At higher altitudes the distribution becomes increasingly perpendicular as the wave-particle interactions heat the ions transverse to the magnetic field. By about $4000 \mathrm{~km}$, the ion distribution function has the typical perpendicularly heated "pancake" shape typical of ion conics forming in the cusp.

This simulation is only meant as an idealized demonstration of PWOM's ability to model conic formation in the cusp. As noted in the model description section, there are a number of uncertainties related the precise form of the wave-heating term and its altitude profile. Additionally, any heating that occurs below the hydrodynamic-kinetic transition is not included; although the boundary could be lowered, or an isotropic heating term could be added to the hydrodynamic model if necessary. Future satellite missions are needed to better constrain the uncertainties associated with the wave inputs.

\subsection{Global Simulation of Cusp Effects}

An important feature of PWOM is that it can follow multiple field lines to reconstruct the full three dimensional solution. This feature is now used to examine the effect of the cusp on the global outflow calculation. To model this scenario, we choose a typical 2-cell convection pattern with which to move the field lines. We then impose an artificial cusp region on the dayside as shown in Figure 7. In this cusp region we turn on waveparticle interactions (with the same heating terms described earlier) and place a monoenergetic beam of precipitating electrons with a mean energy of $100 \mathrm{eV}$ and energy flux of $1 \mathrm{erg} / \mathrm{cm}^{2} / \mathrm{s}$. 896 field lines are then allowed to move around the polar cap entering and leaving the cusp region.

Figure 7 presents the PWOM results at $6000 \mathrm{~km}$ in the top right. The $\mathrm{O}^{+}$and $\mathrm{H}^{+}$ fluxes are shown as well as what percent of the solution is $\mathrm{O}^{+}$. There is clearly an enhanced $\mathrm{O}^{+}$flux associated with the cusp and a more modest $\mathrm{H}^{+}$flux enhancement; a linear scale is used to highlight the peak flux. Moreover, in the vicinity of the cusp the frac- 


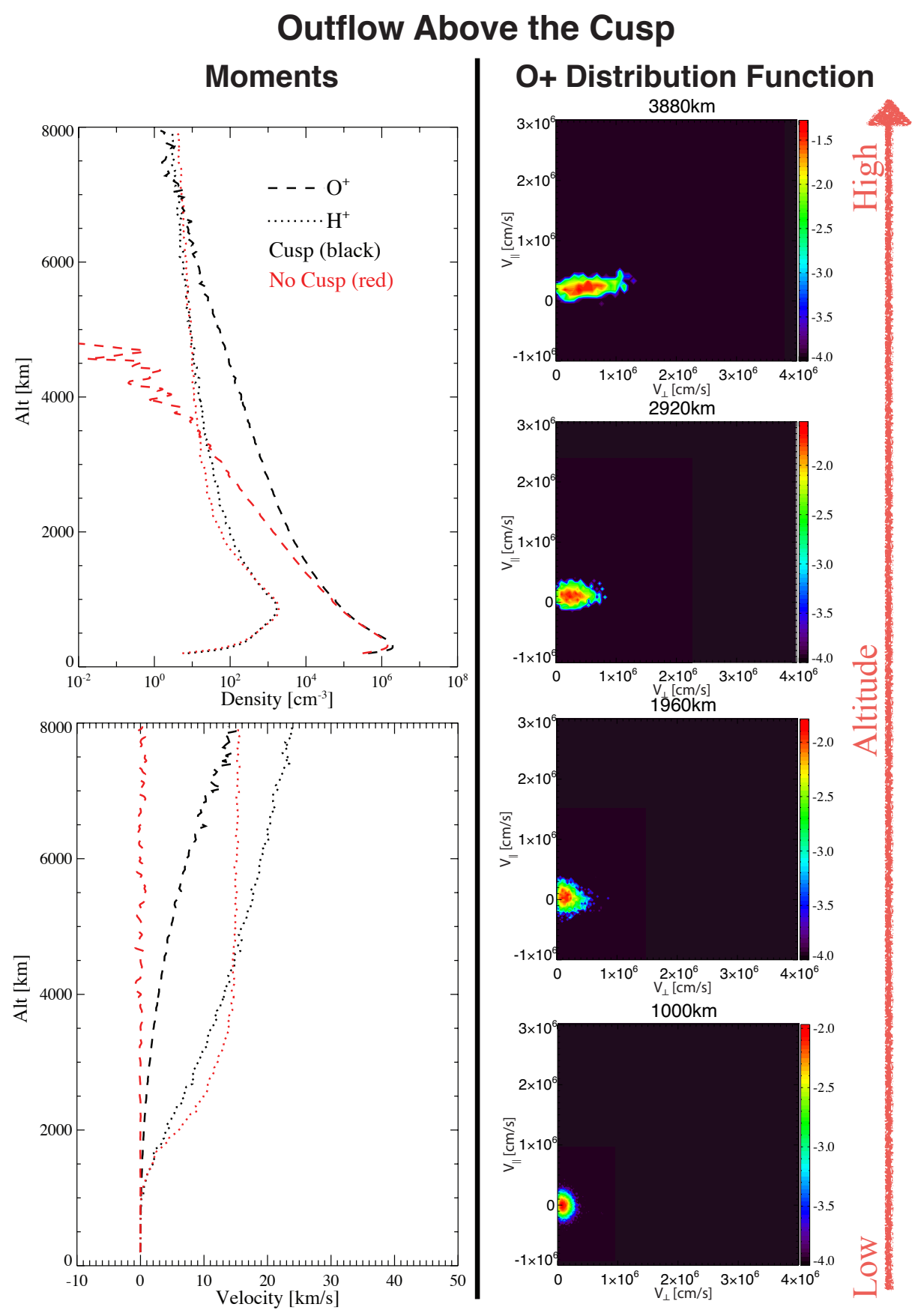

Figure 6. A sunlit cusp field line exposed to soft electron precipitation $\left(1 \mathrm{erg} \mathrm{cm}^{-2} \mathrm{~s}^{-1}\right.$ at $\left.100 \mathrm{eV}\right)$ and wave-particle interactions for 25 minutes. Altitude profiles of density (top) and velocity (bottom) are shown on the left. The solution from Figure 5 is over-plotted in red as a reference. The right panel presents the $\mathrm{O}^{+}$ velocity space distribution function at selected altitudes. These field lines have significant $\mathrm{O}^{+}$available at high altitudes with ion conic formation becoming evident by $4000 \mathrm{~km}$. 


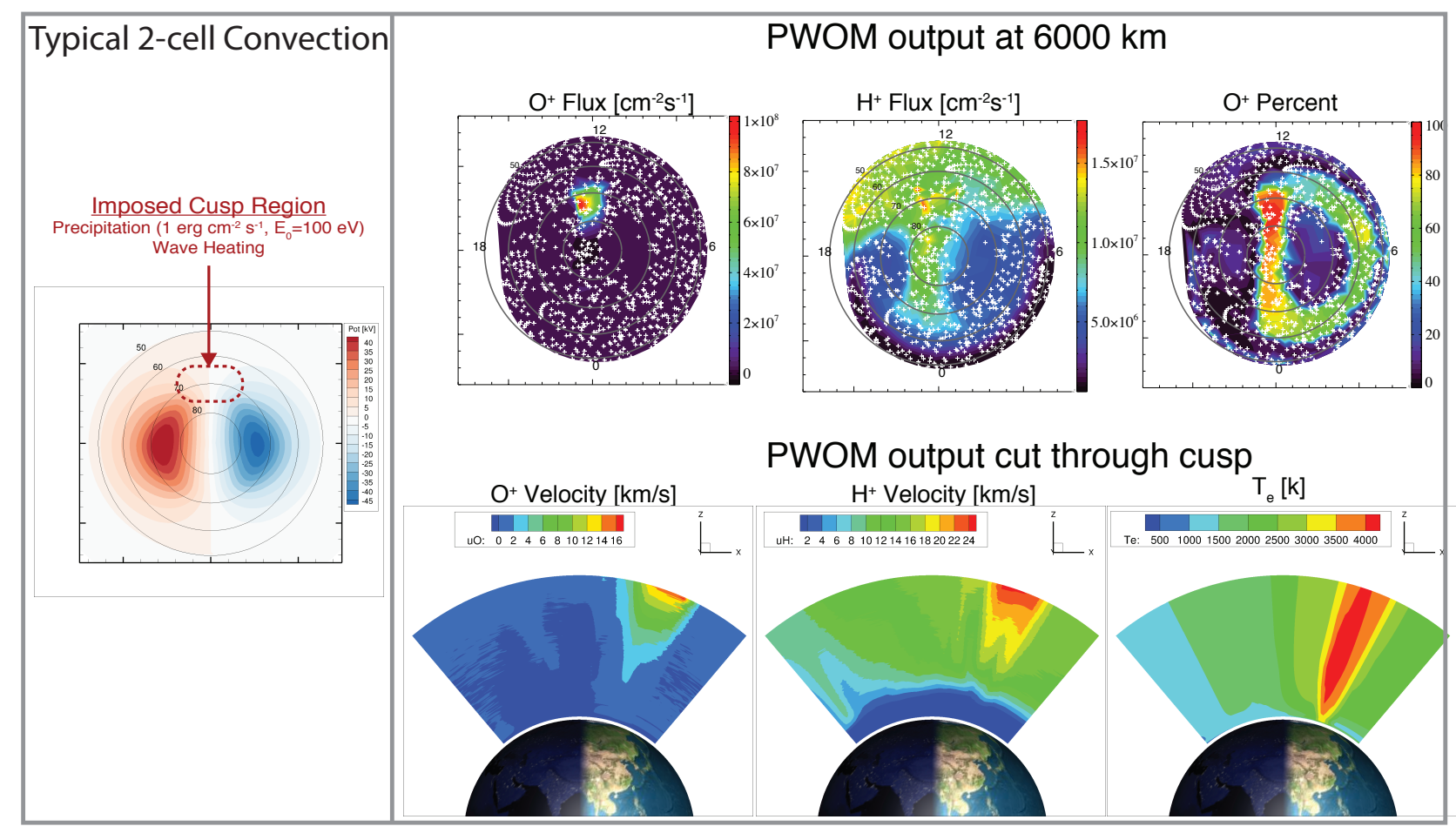

Figure 7. Global polar wind solution using a typical 2-cell convection pattern with an imposed cusp region (shown left). Polar plots at fixed altitude are presented on the top right showing the $\mathrm{O}^{+}$and $\mathrm{H}^{+}$flux as well as the percent $\mathrm{O}^{+}$. The ' + ' symbols indicate field line locations. The lower right presents the $\mathrm{O}^{+}$and $\mathrm{H}^{+}$ velocity and the electron temperature on a plane cut through the cusp.

tion of $\mathrm{O}^{+}$is strongly enhanced. The $\mathrm{O}^{+}$enhancement persists poleward of the cusp as accelerated plasma is convected across the polar region. This enhanced $\mathrm{O}^{+}$fraction at high altitudes over the polar cap, originating in the cusp, is often a source of confusion in observations as the plasma origin cannot be established [e.g., Lockwood et al., 1985].

One unexpected feature in the simulation is that there is enhanced $\mathrm{H}^{+}$flux extending across the polar cap to the night side. In the model, we believe this is due to increased $\mathrm{O}^{+}$ production in the cusp being transported across the polar cap while simultaneously producing $\mathrm{H}^{+}$through accidentally resonant charge exchange. The combination of transport time and reaction time conspire to create additional $\mathrm{H}^{+}$poleward of the cusp which can then be accelerated by the classical polar wind mechanism creating enhanced $\mathrm{H}^{+}$fluxes.

The bottom right of Figure 7 presents the $\mathrm{O}^{+}$and $\mathrm{H}^{+}$velocity as well as the electron temperature on a cut plane through the cusp. These plots illustrate the altitude evolution of the cusp enhancement. Note that the strongest $\mathrm{O}^{+}$and $\mathrm{H}^{+}$velocities are located at higher altitudes and are driven by the WPI. We note the electron temperature is enhanced in the cusp region owing to the addition of soft electron precipitation. Soft electron precipitation in the cusp raises the electron temperature, increase the ionization rate, and results in upwelling of ions which can be further accelerated by WPI. As shown by Barakat and Schunk [1983] and Demars et al. [1996], enhanced electron temperatures are associated with enhanced $\mathrm{O}^{+}$fluxes. The connection between enhanced outflow and electron temperature is also seen in observations [e.g., Abe et al., 1993]. The picture presented here is consistent with these past studies. 
We note that the parameters defining the "artificial" cusp region shown in Figure 7 are chosen to represent very strong cusp effects on the outflow solution. As such the values for precipitation in the cusp region are chosen to appropriate for more active conditions. The $1 \mathrm{erg} / \mathrm{cm}^{2} / \mathrm{s}$ precipitating electron energy flux is half of the value used by Deng et al. [2013], which they justified based on previous studies as appropriate for very strong geomagnetic events. Additionally, the cusp region is imposed between 65 and 75 degrees latitude and extends about 2 hours of local time centered around noon. This cusp region is somewhat exaggerated in latitude relative to the expected cusp size which is roughly 1-4 degrees [Newell and Meng, 1987, 1992]. Therefore, these simulations represent a maximal effect of the cusp on the outflow. Reducing the cusp size would likely reduce the wave heating of ions traversing the cusp region as they would have a reduced residence time in the cusp. We plan to explore the effects of varying the cusp size and the precipitating electron flux in future studies along with other parameters not considered here such as the thermosphere neutral densities and temperatures. However, the present values are adequate for this initial test.

\subsection{Initial Two-Way Coupling of Kinetic Outflow to Multi-Fluid MHD Magneto- sphere}

PWOM was first coupled to BATS-R-US by Glocer et al. [2009b] where the outflow parameters calculated by PWOM are used to set the inner boundary conditions for the magnetosphere. This coupling provides a first-principles specification of the ionospheric source of plasma for the magnetosphere. As none of the model development presented here significantly affects the mechanics of the model coupling, all of the new features of PWOM presented in this study are instantly available to provide a specification of the ionospheric source of plasma to the magnetosphere. In this section we present an initial test of our model improvements in the context of the coupled space environment system. Specifically, this simulation examines the effectiveness of wave-particle interactions above the cusp and auroral region, in supplying $\mathrm{O}^{+}$to the plasmasheet and ring current.

The specific setup of coupled models is illustrated in Figure 8 and described in detail in Glocer et al. [2009a] and Glocer et al. [2013]. For convenience of the reader, we briefly summarize the coupling as follows. The global magnetosphere is represented by the multi-fluid version of the BATS-R-US code [Glocer et al., 2009]. It is coupled to the ionosphere using a height integrated potential solver [Ridley et al., 2004] which combines the field aligned currents from the magnetosphere with the Hall and PEderson conductivities obtained from empirical relations in order to calculate the potential in the polar region. That potential is mapped to the inner boundary of the magnetosphere where it is used to set the transverse velocities. The ionospheric outflow is described by PWOM which takes the polar cap potential and precipitation as inputs. Note the precipitation is inferred from the field-aligned currents [Ridley et al., 2004] and in this case represents only the mono-energetic aurora. The outflow solution is then interpolated onto the BATSR-US inner boundary in order to set the inner boundary face values of the calculation.

The ring current in this calculation is represented using the Comprehensive Inner Magnetosphere (CIMI) Model [Fok et al., 2014]. CIMI takes the magnetic field, electric potential, and plasmasheet boundary conditions as inputs and carries out a bounce averaged kinetic calculation of the ring current distribution function. The resulting pressure and density for each species is provided back to BATS-R-US to correct the MHD values in the inner magnetosphere. A predecessor of this model, the Comprehensive Ring Current Model (CRCM), was fully coupled to BATS-R-US by Glocer et al. [2013]. CIMI uses the same coupling infrastructure, slightly modified to allow coupling multiple ion species to the ring current model; full details of the coupling are available in this previous work. The model coupling is facilitated by the Space Weather Modeling Framework Tóth et al. [2012] with all models exchanging information at a regular cadence. 


\section{Model Coupling: Particle - Fields}

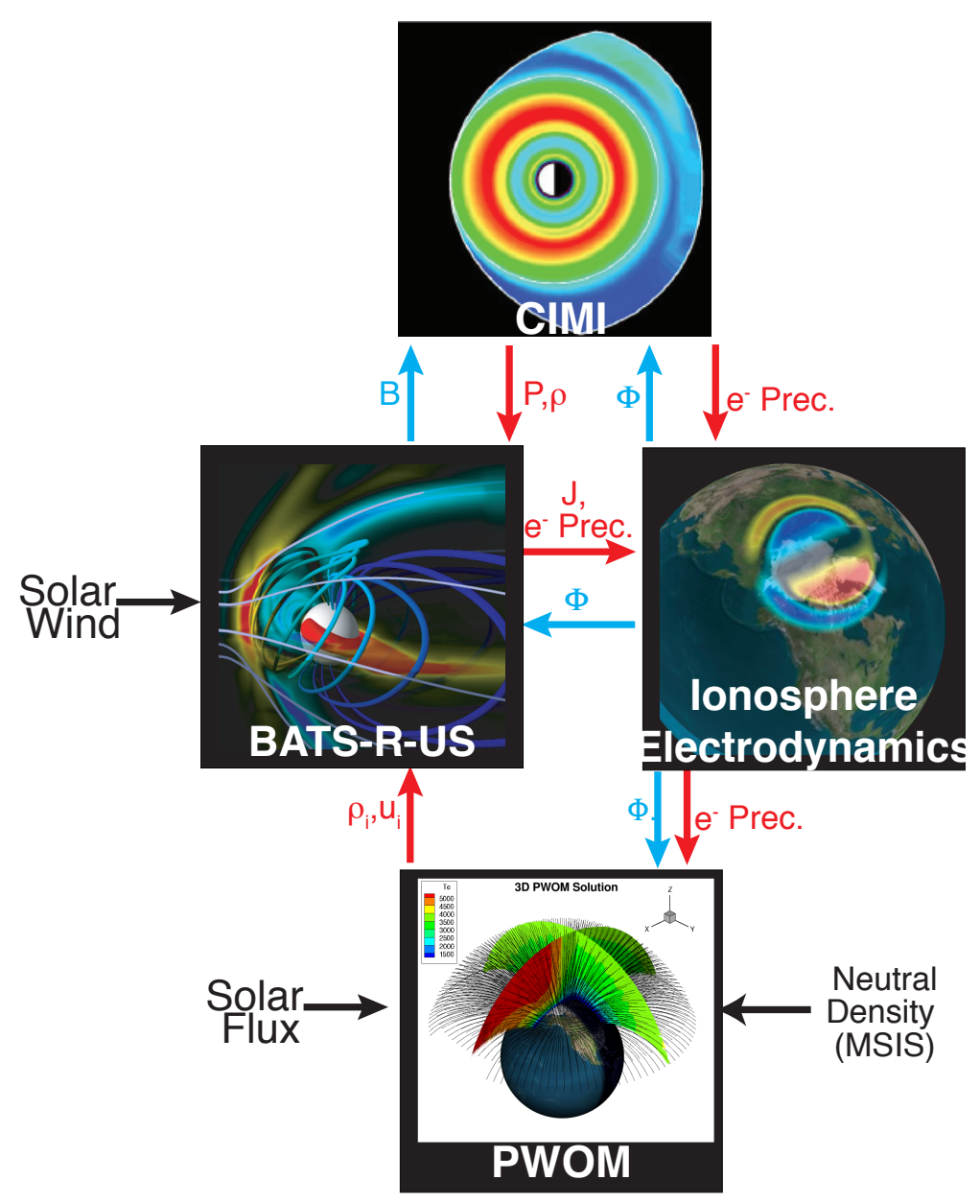

Figure 8. An overview of model coupling illustrating how the outflow fluxes from PWOM are included in the global model of the near-Earth space environment. Particle couplings are shown in red, while couplings based on fields are shown in blue. Model inputs are shown in black.

This article is protected by copyright. All rights reserved. 
As an initial demonstration that the multi-fluid BATS-R-US code can be driven by the kinetic outflow solution from PWOM, we consider the effect of wave-particle interactions in supplying plasma to the magnetosphere. This effect has not been considered in our previous model couplings, as the hydrodynamic only version of PWOM was not capable of studying the effect of transverse wave heating and conic formation. In this initial study, we consider the magnetosphere at equinox driven by typical southward solar wind conditions $\left(B_{Z}=-5 \mathrm{nT}, \mathrm{n}=5 \mathrm{~cm}^{-3}\right.$, and $\left.\mathrm{v}=400 \mathrm{~km} / \mathrm{s}\right)$. The outflow solution is represented by 125 convecting field lines in PWOM, which are coupled every 60s to BATS-R-US. Although PWOM is capable of separately solving the outflow solution in the northern and southern hemispheres, only the northern hemisphere outflow solution is calculated in this simulation. The southern hemisphere is set to mirror the north in order to save computational expense for this first test. The coupled model is run for approximately 5 hours with fixed inputs.

One challenge is in how to represent the spatial distribution and intensity of the wave power in the coupled model. These inputs are important for driving the wave-particle interactions for the outflow calculation. BATS-R-US, like any MHD or multi-fluid MHD model of the magnetosphere, is unable to specify either of these quantities. However, energized outflows are often observed above regions of auroral precipitation. We therefore rely on the particle precipitation, calculated based on an empirical relationship from the field aligned current [Ridley et al., 2004], to provide the spatial specification of where to put the wave power. Specifically, whenever the precipitation exceeds $1 \mathrm{erg} / \mathrm{cm}^{2} / \mathrm{s}$ we turn on the wave-particle interaction terms specified by Barakat and Barghouthi [1994] for the cusp and aurora. While this approach is a reasonable method for obtaining the spatial distribution of the waves, the intensity is still only a typical value and not modulated by the inputs. An alternative approach would be to follow the example of Varney et al. [2016] and use Alfvenic Poynting flux from the global magnetosphere model to specify wave intensity. Nevertheless, the simple prescription for the wave-power and distribution described above is sufficient for the present study.

Figure 9 shows the resulting magnetospheric composition and field lines in the GSM $y=0$ plane at various times between approximately 3 and 4 hours of simulation time. During this time the lobes of the magnetosphere are enriched with ionospheric $\mathrm{O}^{+}$. The ionospheric plasma fills the lobes and lands in the tail near the reconnection site where it has a significant impact on the magnetotail stability. Between 3 and 4 hours a number of plasmoids form and the x-point moves around between about $15 R_{e}$ down tail to as much as $35 R_{e}$.

Figure 10 shows the corresponding PWOM solution at 4 hours of simulated time with the ionosphere electrodynamics solution at the bottom of the figure. We selected an altitude of $6000 \mathrm{~km}$ at which to examine the outflow. A number of interesting features are visible in the solution. First, the ion density, particularly for $\mathrm{O}^{+}$, is skewed by the convection pattern. Note that a skewed, or asymmetric, convection pattern can result even for pure southward IMF as the ring current drift causes the inner magnetospheric pressure to peak in the pre-midnight sector which in turn affects the FACs and the associated polar cap potential. Another interesting feature of the outflowing solution is that the $\mathrm{O}^{+}$velocity is elevated following the auroral oval indicating an auroral wind in our simulation. This is expected as the wave heating terms are included in a phenomenological manner following the precipitation (described above). It is likewise notable that while the $\mathrm{O}^{+}$outflow is more organized by the particle precipitation, the $\mathrm{H}^{+}$is more organized by the the solar illumination. Nevertheless, both species of outflowing plasma have their strongest fluxes on the dayside under illuminated conditions.

The last point has some broader implications and warrants further discussion. While the $\mathrm{O}^{+}$velocity is elevated everywhere around the auroral oval, the outflowing flux is much stronger on the dayside. This simulation feature can be understood when remembering that the wave-heating accelerating the plasma can create large velocities, but it can- 

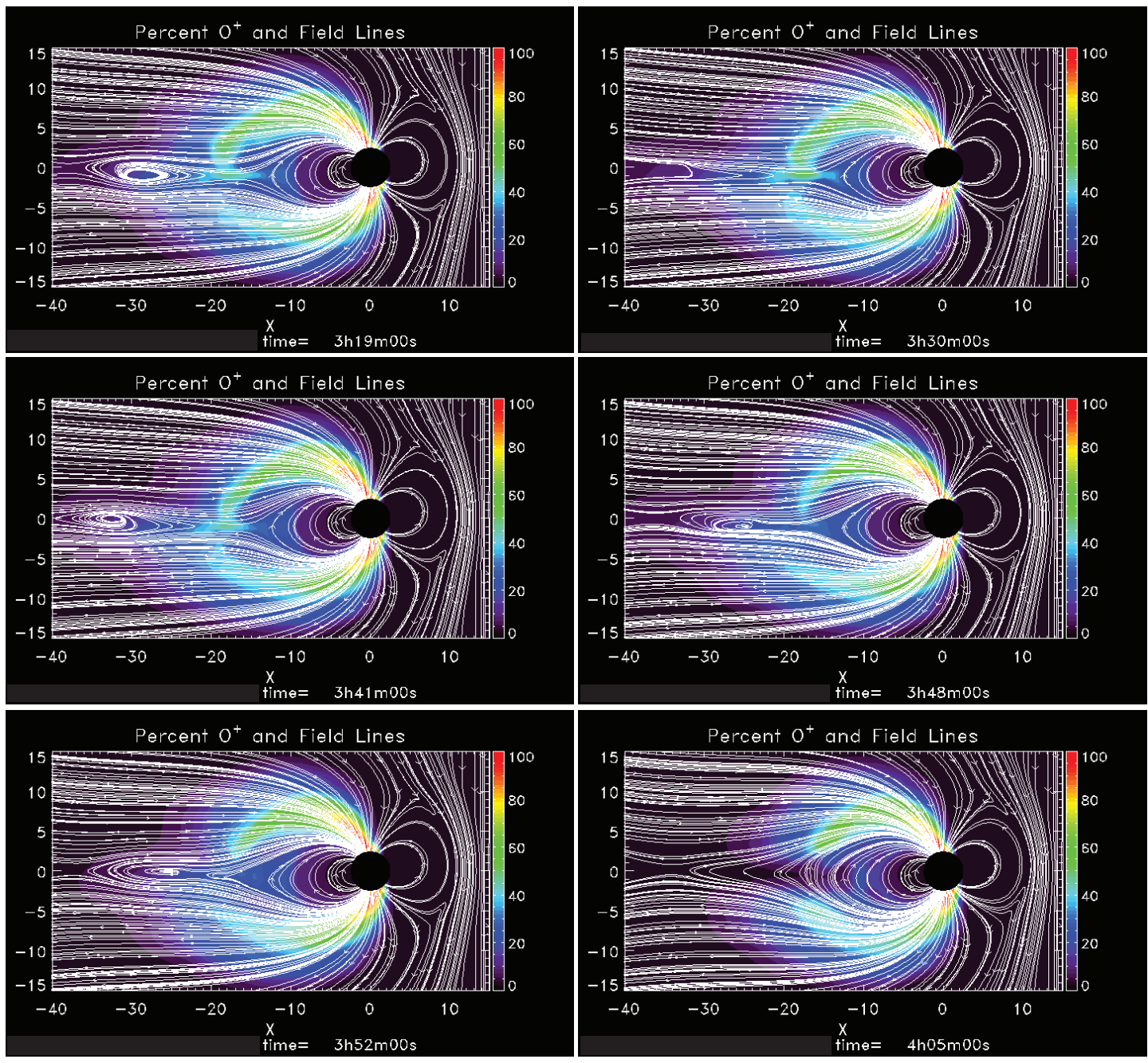

Figure 9. Color contours of composition and field lines in the $y=0$ GSM plane at different times showing 


\section{PWOM Output at $6000 \mathrm{~km}-$ Time $=4 \mathrm{hr}$}
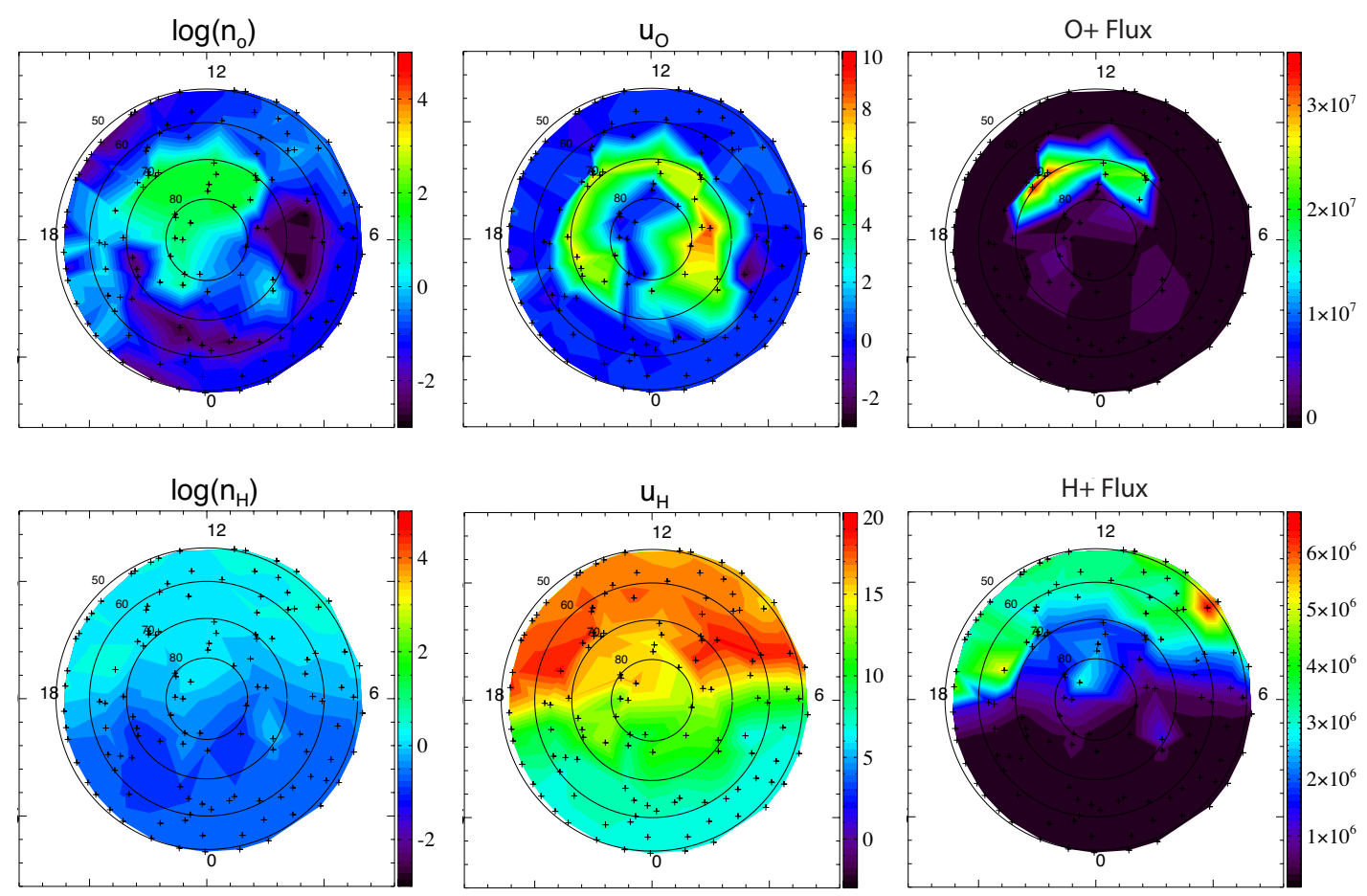

\section{Ionosphere Electrodynamics Output - Time $=4 \mathrm{hr}$}
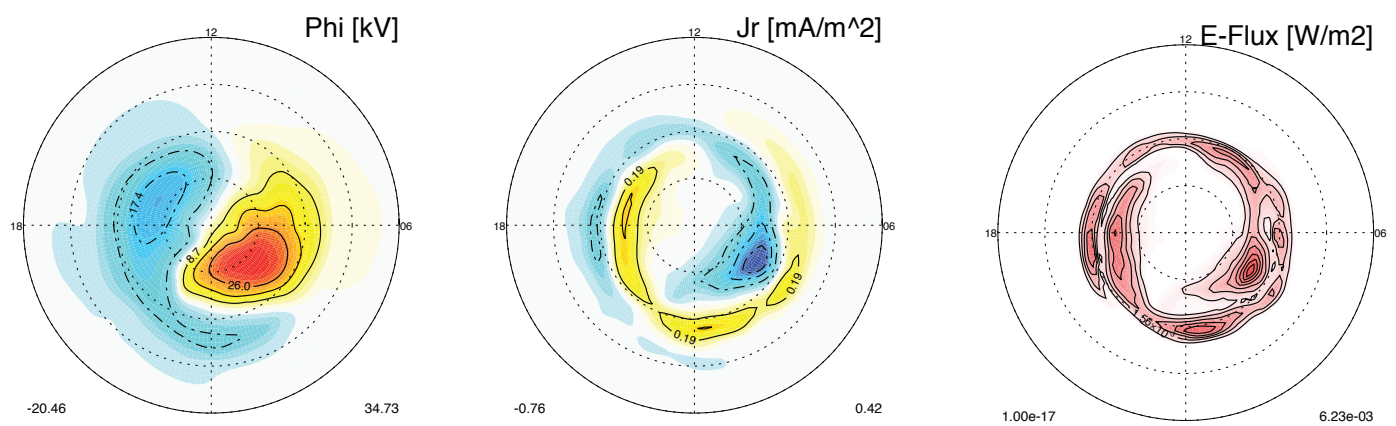

Figure 10. Top panel presents PWOM output looking down at the polar cap of density, velocity, and flux for $\mathrm{O}^{+}$and $\mathrm{H}^{+}$at $6000 \mathrm{~km}$ altitude. The sunward direction is at the top of the plot and midnight at the bottom. The bottom panel shows the ionospheric electrodynamics solution (potential, FAC, and auroral energy flux) at the base of the field line. 
not produce ions to accelerate. In our model, the $\mathrm{O}^{+}$is mainly produced from either photoionization or impact ionization from energetic electrons. As the precipitation from the ionosphere electrodynamics model only provides hard auroral precipitation, most of the associated impact ionization is produced in the E region where it contributes to the conductance but at too low an altitude to contribute to the outflowing plasma. Therefore, ion production due to photoionization is critical to defining the amount of plasma available for energization by wave processes and sets an important limit on the ion flux that can be generated by wave-particle interactions. As a result, identical wave heating terms all around the auroral oval are able to produce high velocities everywhere, but larger fluxes are found on the dayside where most of the ion production occurs. As a caveat, it is important to keep in mind that a number of factors can influence this picture. The inclusion of soft electron precipitation that enhances ion production and energy deposition in the Fregion could result in stronger outflows on both the day and nightside. Likewise, periods of enhanced convection that can transport ions produced on the dayside to the nightside, where they can be accelerated by wave-particle interactions in auroral region, can also enhance nightside outflow.

A clear implication of the this discussion is that the outflowing plasma solution depends on the local conditions of illumination, particle precipitation, wave-parameters, and the time history of the flux tube. This is problematic when trying to specify an empirical formula to represent the outflowing plasma as has been done in a number of studies to date. Indeed, most of these studies rely on a useful empirical formula presented by Strangeway et al. [2005] that relates electron precipitation and Poynting flux to total ion flux. While this formula is derived based entirely on outflow observations in the cusp, it is frequently applied throughout the entire high latitude region. In light of the present results, one should be cautious when applying an empirical relationship based solely on cusp data to other high latitude locations as the illumination and precipitation characteristics in the dayside cusp and nightside aurora are quite different.

The distribution of the outflow also informs our understanding of the tail dynamics. Wiltberger et al. [2010] considered a multi-fluid MHD simulation with an imposed cusp like outflow occurring on the dayside. They found that the outflow lands near the reconnection site and the location of the x-line can move significantly. In contrast, Brambles et al. [2011] used the same multi-fluid MHD code with an empirically specified boundary using a version of the relationship of Strangeway et al. [2005]. They found strong outflow on the auroral field lines which stretch the closed field lines by overloading them with ionospheric plasma and lead to periodic sawtooth events. The tail dynamics observed in Figure 9 are driven more by dayside outflow and are thus more similar to the case described by Wiltberger et al. [2010]. Indeed, for the reasons discussed above, it is possible that large outflow fluxes on auroral field lines, based on an empirical formula derived for the dayside cusp, may be overestimated. We note, however, that the sawtooth behavior in Brambles et al. [2011] exhibited strong dependence on the upstream driving conditions, e.g., sawtooth oscillations occur only under CME storm type conditions with $\mathrm{B}_{z}=-10 \mathrm{nT}$, $\mathrm{V}_{x}=600 \mathrm{~km} / \mathrm{s}$. However, the coupled simulation results presented in this section were driven by more moderate conditions $\left(\mathrm{B}_{z}=-5 \mathrm{nT}, \mathrm{V}_{x}=400 \mathrm{~km} / \mathrm{s}\right)$. It is possible that the picture in this section could change under more intense driving condition. For example, more intense driving conditions may result in stronger convection which could increase the transport of plasma from the dayside to the nightside polar cap thereby increasing the plasma available for acceleration on auroral field lines. Further investigation is needed to understand what conditions could lead to strong outflow fluxes, not just high velocities, on auroral fieldlines in order to better understand and test the hypothesized connection between outflow and sawteeth events.

Finally, we examine the contribution of outflow to the ring current under these idealized conditions. Figure 11 presents the ring current $\mathrm{O}^{+}$and $\mathrm{H}^{+}$pressures 4 hours into the simulation. As expected, the ring current pressure exhibits a peak in the premidnight 


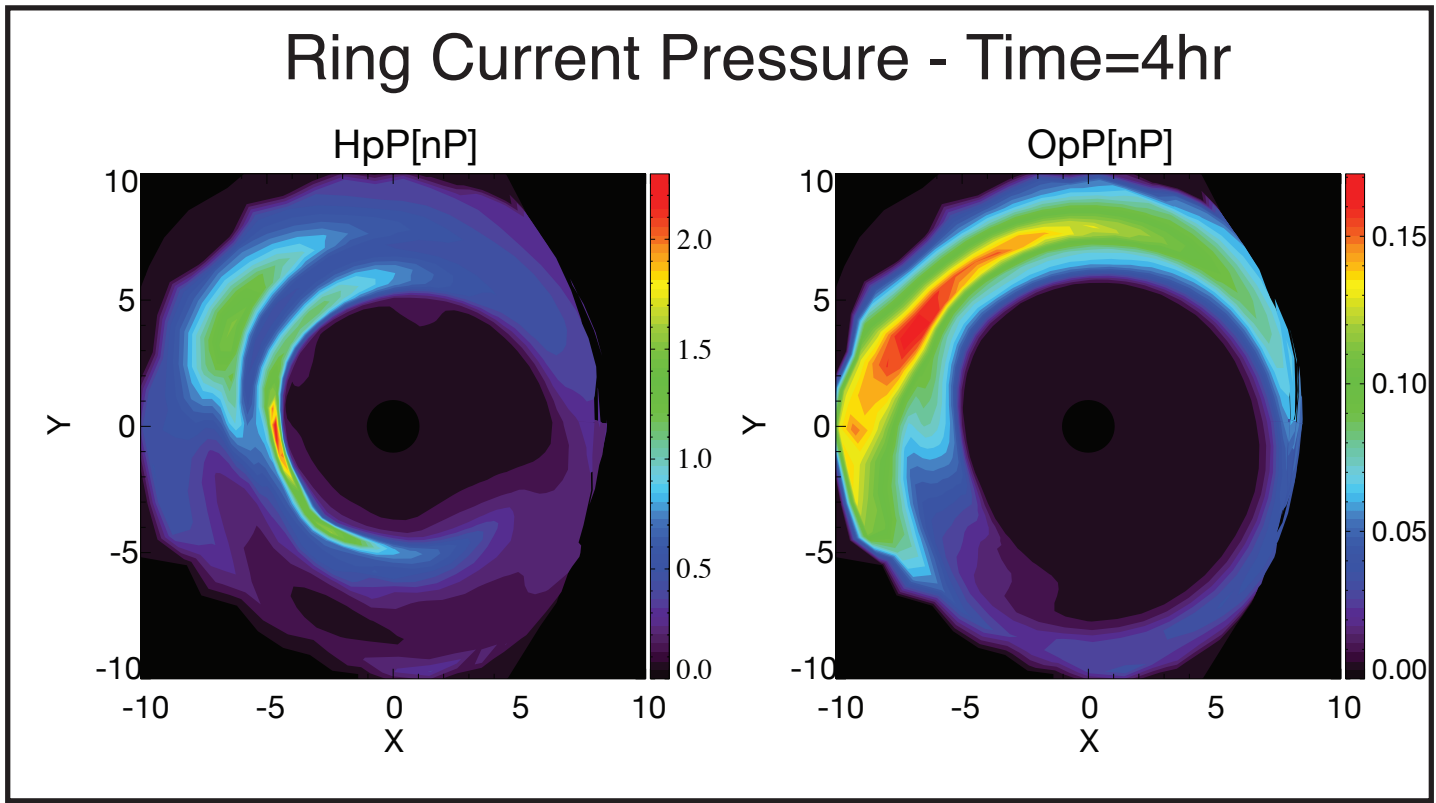

Figure 11. The output of the ring current solution on the minimum B surface from CIMI including, the $\mathrm{H}^{+}$(left) and $\mathrm{O}^{+}$(right) pressure. All output is at 4 hours into the simulation. Note that $\mathrm{O}^{+}$accounts for approximately $10 \%$ of the peak ring current pressure at this time.

sector owing to the energy dependent ion drifts. We also find that $\mathrm{O}^{+}$accounts for approximately $10 \%$ of the peak ring current pressure at this time. Such a value is typical for periods of low geomagnetic activity. This provides a reasonable first demonstration that the CIMI model is capable of working with the SWMF and coupling with the multi-fluid MHD version of BATS-R-US.

The primary focus of the present simulation is to demonstrate the ability to use the new kinetic ion features of PWOM in a global geospace simulation. The success of this initial simulation and the reasonableness of the results indicate that it is now possible to include kinetic polar wind ions in global simulations at reasonable computational expense. The examination of a wider range of geomagnetic activity is left to future studies.

\section{Conclusions}

We presented new features of the Polar Wind Outflow Model (PWOM) that enable the inclusion of kinetic ions, wave-particle interactions, and suprathermal electron effects in a three-dimensional global outflow solution. This modeling approach causally treats most major outflow mechanisms using a first principles approach. The new model development combines a multi-fluid hydrodynamic approach at lower altitudes with a hybridDSMC, or Mac-PIC, solution at high altitudes in a two-way coupled manner. The interface between the two modeling descriptions occurs in the transition region where both modeling approaches are physically appropriate descriptions. As a result, the outflow modeling approach is valid at all altitudes.

We note that this approach is similar in concept to two existing codes: the Dynamic Fluid-Kinetic (DyFK) model [Estep et al., 1999; Zeng and Horwitz, 2007] and General Polar Wind (GPW) model [Barakat and Barghouthi, 1994]. However, the implementation in PWOM, presented here, has a number of new advantageous features. First, PWOM in- 
cludes the effects of suprathermal electrons in the global solution by using the GLOW model, allowing an efficient treatment of the effects of photoelectrons, auroral electrons, and secondary electrons on the outflowing solution. Second, the use of variably weighted particles and particle rezoning (also known as particle splitting and joining) helps to increase robustness and reduce particle noise to a certain extent. Third, PWOM uses a combination of distributed memory (with MPI) and shared memory (with OpenMP parallelism) to speed up the computation. By putting each field line on a separate MPI process and using 5 OpenMP threads on each MPI process to speed up the particle work, a global outflow solution including kinetic ions can be obtained in the same amount of time as it takes to obtain a pure hydrodynamic solution with no OpenMP acceleration. Finally, PWOM has the benefit of being fully integrated into the SWMF to support global studies of outflow and the feedback of the rest of geospace on the outflow. Combined with the improved parallelization scheme, this last point means that global kinetic outflow solutions are computationally feasible as part of a coupled geospace system.

To test the model improvements, we considered four problems in order of increasing complexity starting with single field line solutions and progressing to multi-field line global solutions. The simplest problem was the sunlight polar field line where we compared the hydrodynamic only solution with the combined solution with kinetic ions at high altitudes. We found that the kinetic and hydrodynamic $\mathrm{H}^{+}$solutions are largely similar, but that larger differences are seen in the $\mathrm{O}^{+}$. We then considered a sunlit cusp field line with soft electron precipitation and wave-particle interactions representing ion cyclotron resonant heating included. This case demonstrates that the model can be used to study the altitude evolution and formation of ion conic distributions.

Two multi-field line global outflow simulations were also considered. The first was a simplified case of two-cell convection with an artificially imposed cusp region with soft electron precipitation and wave heating. This simulation found enhanced outflow in the cusp with the convection pulling enhanced upflowing plasma over the polar cap. Understanding the contribution of cusp accelerated plasma to the polar cap population is a common observational problem (see e.g. Yau et al. [2007]). We also observed $\mathrm{H}^{+}$flux enhancements continueing poleward of the cusp which can possibly be explained by the interplay between convection and charge exchange timescales. Finally, we considered an idealized simulation where the outflow solution from PWOM is coupled into the SWMF where it supplies plasma to the magnetosphere, while simultaneously taking magnetospheric inputs. In addition to the magnetosphere, polar wind and ionospheric components, the simulation also included the CIMI inner magnetosphere model. This simulation coupled the outflow dynamically with the magnetosphere and demonstrated the feasibility of including kinetic ions and suprathermal electron effects in the global outflow solution. We found that the outflow occurs preferentially on the dayside, even though wave heating occurs everywhere around the auroral oval. The outflow fills the lobes and lands near the reconnection site in the tail where it affects the tail stability. For the conditions considered, there is only a modest contribution of $\mathrm{O}^{+}$to the total ring current pressure.

The present work described significant new developments of the PWOM code and presented initial test cases. There remain, however, a number of interesting questions that are deferred to future studies. Notably, there is a great deal of focus on the $\mathrm{O}^{+}$outflow as oxygen is a clear indicator of an ionospheric source of plasma. However, the simulations show a significant amount of polar wind $\mathrm{H}^{+}$coming out over a large area of the high latitude region. Currently we do not distinguish between ionospheric and solar wind protons in the magnetosphere. The relative influence of ionospheric $\mathrm{H}^{+}$on magnetospheric processes is an important topic that will be evaluated in future studies. Likewise, the evaluation of the model for real events, and the sensitivity to assumptions regarding the wave heating are deferred to future studies. 


\section{Acknowledgments}

A. Glocer and M.-C. Fok acknowledge support from the NASA Strategic Capability program (WBS 936723.02.01.09.21) and the Heliophysics Grand Challenge Research program (WBS 791726.02.04.01.83). G. Toth's effort was supported by the NSF PREEVENTS grant 1663800. Resources supporting this work were provided by the NASA High-End Computing (HEC) Program through the NASA Advanced Supercomputing (NAS) Division at Ames Research Center. All modeling tools described in publication are available online through the Univeristy of Michigan for download and will also be made available for use at the Community Coordinated Modeling Center (CCMC). The model output data are the numerical information provided in figures; these are produced by applying the numerical model whose availability is given above. Alternatively, numerical information associated with these figures is available by contacting A. Glocer. The authors would also like to thank J. Dorelli for helpful discussions and S. Solomon for making the GLOW model available.

\section{References}

Abe, T., B. A. Whalen, A. W. Yau, S. Watanabe, E. Sagawa, and K. I. Oyama (1993), Altitude profile of the polar wind velocity and its relationship to ionospheric conditions, Geophys. Res. Lett., 20, 2825-2828, doi:10.1029/93GL02837.

Axford, W. I. (1968), The polar wind and the terrestrial helium budget, J. Geophys. Res., $73,68,55$.

Banks, P. M., and T. E. Holzer (1968), The Polar Wind, J. Geophys. Res., 73, 6846-6854, doi:10.1029/JA073i021p06846.

Banks, P. M., and A. F. Nagy (1970), Concerning the Influence of Elastic Scattering Upon Photoelectron Transport and Escape, J. Geophys. Res., 75, 1902-1910, doi: 10.1029/JA075i010p01902.

Barakat, A. R., and I. A. Barghouthi (1994), The effect of wave-particle interactions on the polar winds $\mathrm{O}(+)$, Geophys. Res. Lett., 21, 2279-2282.

Barakat, A. R., and R. W. Schunk (1983), O(+) ions in the polar wind, J. Geophys. Res., 88, 7887-7894, doi:10.1029/JA088iA10p07887.

Barakat, A. R., and R. W. Schunk (2006), A three-dimensional model of the generalized polar wind, Journal of Geophysical Research: Space Physics, 111(A12), n/a-n/a, doi: 10.1029/2006JA011662, a12314.

Barakat, A. R., I. A. Barghouthi, and R. W. Schunk (1995), Double-hump $\mathrm{H}^{+}$velocity distribution in the polar wind, Geophys. Res. Lett., 22, 1857-1860, doi: 10.1029/95GL01519.

Bilitza, D., K. Rawer, L. Bossy, I. Kutiev, K.-I. Oyama, R. Leitinger, and E. Kazimirovsky (1990), International reference ionosphere 1990.

Brambles, O. J., W. Lotko, B. Zhang, M. Wiltberger, J. Lyon, and R. J. Strangeway (2011), Magnetosphere Sawtooth Oscillations Induced by Ionospheric Outflow, Science, 332(6034), 1183-1186.

Chappell, C. R., T. E. Moore, and J. H. Waite (1987), The ionosphere as a fully adequate source of plasma for the earth's magnetosphere, J. Geophys. Res., 92, 5896-5910.

Crew, G. B., T. Chang, J. M. Retterer, W. K. Peterson, and D. A. Gurnett (1990), Ion cyclotron resonance heated conics - Theory and observations, J. Geophys. Res., 95, 3959.

Demars, H. G., A. R. Barakat, and R. W. Schunk (1996), Effect of centrifugal acceleration on the polar wind, Journal of Geophysical Research: Space Physics, 101(A11), 24,56524,571, doi:10.1029/96JA02234.

Deng, Y., T. J. Fuller-Rowell, A. J. Ridley, D. Knipp, and R. E. Lopez (2013), Theoretical study: Influence of different energy sources on the cusp neutral density enhancement, Journal of Geophysical Research: Space Physics, 118(5), 2340-2349, doi: $10.1002 /$ jgra.50197. 
Dessler, A. J., and P. A. Cloutier (1969), Discussion of letter by peter m. banks and thomas e. holzer, âĂŸthe polar windâĂŹ, Journal of Geophysical Research, 74(14), 3730-3733, doi:10.1029/JA074i014p03730.

Donahue, T. M. (1971), Polar ion flow: Wind or breeze?, Reviews of Geophysics, 9(1), 19, doi:10.1029/RG009i001p00001.

Estep, G. M., J. L. Horwitz, Y.-J. Su, P. G. Richards, G. R. Wilson, and D. G. Brown (1999), A dynamic fluid-kinetic (dyfk) model for ionosphere-magnetosphere plasma transport: Effects of ionization and thermal electron heating by soft electron precipitation, Terrestrial, Atmospheric and Oceanic Sciences, 10(3), 491-510.

Fok, M., T. E. Moore, P. C. Brandt, D. C. Delcourt, S. P. Slinker, and J. A. Fedder (2006), Impulsive enhancements of oxygen ions during substorms, Journal of Geophysical Research (Space Physics), 111, 10,222-+, doi:10.1029/2006JA011839.

Fok, M.-C., N. Y. Buzulukova, S.-H. Chen, A. Glocer, T. Nagai, P. Valek, and J. D. Perez (2014), The comprehensive inner magnetosphere-ionosphere model, Journal of Geophysical Research: Space Physics, 119(9), 7522-7540, doi:10.1002/2014JA020239, 2014JA020239.

Garcia, K. S., V. G. Merkin, and W. J. Hughes (2010), Effects of nightside o+ outflow on magnetospheric dynamics: Results of multifluid mhd modeling, Journal of Geophysical Research: Space Physics, 115(A12), n/a-n/a, doi:10.1029/2010JA015730, a00J09.

Glocer, A., T. I. Gombosi, G. Toth, K. C. Hansen, A. J. Ridley, and A. Nagy (2007), Polar wind outflow model: Saturn results, J. Geophys. Res., 112, doi:10.1029/2006JA011755.

Glocer, A., G. Toth, T. Gombosi, and D. Welling (2009a), Modeling ionospheric outflows and their impact on the magnetosphere, initial results, J. Geophys. Res., 114(A05216), doi:10.1029/2009JA014053.

Glocer, A., G. Toth, M. Fok, T. Gombosi, and M. Liemohn (2009b), Integration of the radiation belt environment model into the space weather modeling framework, Journal of Atmospheric and Solar-Terrestrial Physics, 71, 1653 - 1663, doi: 10.1016/j.jastp.2009.01.003.

Glocer, A., G. Tóth, Y. Ma, T. Gombosi, J.-C. Zhang, and L. M. Kistler (2009), Multifluid Block-Adaptive-Tree Solar wind Roe-type Upwind Scheme: Magnetospheric composition and dynamics during geomagnetic storms - Initial results, Journal of Geophysical Research (Space Physics), 114(A13), A12203, doi:10.1029/2009JA014418.

Glocer, A., N. Kitamura, G. Toth, and T. Gombosi (2012), Modeling solar zenith angle effects on the polar wind, Journal of Geophysical Research: Space Physics, 117(A4), n/a-n/a, doi:10.1029/2011JA017136, a04318.

Glocer, A., M. Fok, X. Meng, G. Toth, N. Buzulukova, S. Chen, and K. Lin (2013), CRCM + BATS-R-US two-way coupling, Journal of Geophysical Research (Space Physics), 118, 1635-1650, doi:10.1002/jgra.50221.

Glocer, A., G. Khazanov, and M. Liemohn (2017), Photoelectrons in the quiet polar wind, Journal of Geophysical Research: Space Physics, 122(6), 6708-6726, doi: 10.1002/2017JA024177, 2017JA024177.

Gombosi, T. I., and A. Nagy (1989), Time-dependent modeling of field aligned currentgenerated ion transients in the polar wind, J. Geophys. Res., 94, 359-369.

Gurnett, D. A., R. L. Huff, J. D. Menietti, J. L. Burch, J. D. Winningham, and S. D. Shawhan (1984), Correlated low-frequency electric and magnetic noise along the auroral field lines, Journal of Geophysical Research: Space Physics, 89(A10), 8971-8985, doi:10.1029/JA089iA10p08971.

Holzer, T. E., J. A. Fedder, and P. M. Banks (1971), A comparison of kinetic and hydrodynamic models of an expanding ion-exosphere, Journal of Geophysical Research, 76(10), 2453-2468, doi:10.1029/JA076i010p02453.

Ilie, R., R. M. Skoug, P. Valek, H. O. Funsten, and A. Glocer (2013), Global view of inner magnetosphere composition during storm time, Journal of Geophysical Research: Space Physics, 118(11), 7074-7084, doi:10.1002/2012JA018468. 
Khazanov, G. V., M. W. Liemohn, and T. E. Moore (1997), Photoelectron effects on the self-consistent potential in the collisionless polar wind, J. Geophys. Res., 102, 75097522, doi:10.1029/96JA03343.

Kozyra, J. U., T. E. Cravens, A. F. Nagy, E. G. Fontheim, and R. S. B. Ong (1984), Effects of energetic heavy ions on electromagnetic ion cyclotron wave generation in the plasmapause region, J. Geophys. Res., 89, 2217-2233, doi:10.1029/JA089iA04p02217.

Lapenta, G. (2002), Particle rezoning for multidimensional kinetic particle-incell simulations, Journal of Computational Physics, 181(1), 317 - 337, doi: https://doi.org/10.1006/jcph.2002.7126.

Lemaire, J., and M. Scherer (1970), Model of the polar ion-exosphere, Planetary and Space Science, 18(1), 103 - 120, doi:http://dx.doi.org/10.1016/0032-0633(70)90070-X.

Lemaire, J., and M. Scherer (1973), Kinetic models of the solar and polar winds, Reviews of Geophysics, 11(2), 427-468, doi:10.1029/RG011i002p00427.

Lennartsson, W., R. D. Sharp, E. G. Shelley, R. G. Johnson, and H. Balsiger (1981), Ion composition and energy distribution during 10 magnetic storms, J. Geophys. Res., 86, 4628-4638, doi:10.1029/JA086iA06p04628.

Lockwood, M., J. H. Waite, T. E. Moore, J. F. E. Johnson, and C. R. Chappell (1985), A new source of suprathermal o+ ions near the dayside polar cap boundary, Journal of Geophysical Research: Space Physics, 90(A5), 4099-4116, doi: 10.1029/JA090iA05p04099.

Marubashi, K. (1970), Escape of the polar-ionospheric plasma into the magnetospheric tail., Tech. rep., Tokyo Univ.

Miller, R. H., and M. R. Combi (1994), A Coulomb collision algorithm for weighted particle simulations, Geophys. Res. Lett., 21, 1735-1738.

Nagy, A. F., and P. M. Banks (1970), Photoelectron fluxes in the ionosphere, JGR, 75, 6260.

Nanbu, K., and S. Yonemura (1998), Weighted particles in coulomb collision simulations based on the theory of a cumulative scattering angle, Journal of Computational Physics, 145(2), 639 - 654, doi:https://doi.org/10.1006/jcph.1998.6049.

Newell, P., and C.-I. Meng (1992), Mapping the dayside ionosphere to the magnetosphere according to particle precipitation characteristics, Geophys. Res. Lett., 19, 609.

Newell, P. T., and C.-I. Meng (1987), Cusp width and bz : Observations and a conceptual model, Journal of Geophysical Research: Space Physics, 92(A12), 13,673-13,678, doi: 10.1029/JA092iA12p13673.

Nosé, M., S. Taguchi, K. Hosokawa, S. P. Christon, R. W. McEntire, T. E. Moore, and M. R. Collier (2005), Overwhelming $\mathrm{O}^{+}$contribution to the plasma sheet energy density during the October 2003 superstorm: Geotail/EPIC and IMAGE/LENA observations, Journal of Geophysical Research (Space Physics), 110, 9, doi: 10.1029/2004JA010930.

Retterer, J. M., T. Chang, G. B. Crew, J. R. Jasperse, and J. D. Winningham (1987), Monte Carlo modeling of ionospheric oxygen acceleration by cyclotron resonance with broad-band electromagnetic turbulence, Physical Review Letters, 59, 148-151, doi: 10.1103/PhysRevLett.59.148.

Ridley, A., T. Gombosi, and D. Dezeeuw (2004), Ionospheric control of the magnetosphere: conductance, Annales Geophysicae, 22, 567-584.

Shay, M. A., and M. Swisdak (2004), Three-Species Collisionless Reconnection: Effect of $\mathrm{O}^{+}$on Magnetotail Reconnection, Physical Review Letters, 93(17), 175,001-+, doi: 10.1103/PhysRevLett.93.175001.

Solomon, S. C. (2017), Global modeling of thermospheric airglow in the far ultraviolet, Journal of Geophysical Research: Space Physics, 122(7), 7834-7848, doi: 10.1002/2017JA024314, 2017JA024314.

Solomon, S. C., P. B. Hays, and V. J. Abreu (1988), The auroral 6300 ÃĚ emission: Observations and modeling, Journal of Geophysical Research: Space Physics, 93(A9), 9867-9882, doi:10.1029/JA093iA09p09867. 
Strangeway, R. J., R. E. Ergun, Y.-J. Su, C. W. Carlson, and R. C. Elphic (2005), Factors controlling ionospheric outflows as observed at intermediate altitudes, Journal of Geophysical Research (Space Physics), 110, 3221, doi:10.1029/2004JA010829.

Takizuka, T., and H. Abe (1977), A binary collision model for plasma simulation with a particle code, Journal of Computational Physics, 25(3), 205 - 219, doi: https://doi.org/10.1016/0021-9991(77)90099-7.

Tóth, G., B. van der Holst, I. V. Sokolov, D. L. de Zeeuw, T. I. Gombosi, F. Fang, W. B. Manchester, X. Meng, D. Najib, K. G. Powell, Q. F. Stout, A. Glocer, Y.-J. Ma, and M. Opher (2012), Adaptive numerical algorithms in space weather modeling, Journal of Computational Physics, 231, 870-903, doi:10.1016/j.jcp.2011.02.006.

Varney, R. H., M. Wiltberger, B. Zhang, W. Lotko, and J. Lyon (2016), Influence of ion outflow in coupled geospace simulations: 1. physics-based ion outflow model development and sensitivity study, Journal of Geophysical Research: Space Physics, 121(10), 9671-9687, doi:10.1002/2016JA022777, 2016JA022777.

Welling, D. T., and M. W. Liemohn (2014), Outflow in global magnetohydrodynamics as a function of a passive inner boundary source, Journal of Geophysical Research: Space Physics, 119(4), 2691-2705, doi:10.1002/2013JA019374.

Welling, D. T., V. K. Jordanova, S. G. Zaharia, A. Glocer, and G. Toth (2011), The effects of dynamic ionospheric outflow on the ring current, Journal of Geophysical Research (Space Physics), 116, A00J19, doi:10.1029/2010JA015642.

Welling, D. T., A. R. Barakat, J. V. Eccles, R. W. Schunk, and C. R. Chappell (2016), Coupling the Generalized Polar Wind Model to Global Magnetohydrodynamics, pp. 179194, John Wiley \& Sons, Inc., doi:10.1002/9781119066880.ch14.

Wiltberger, M., W. Lotko, J. G. Lyon, P. Damiano, and V. Merkin (2010), Influence of cusp o+ outflow on magnetotail dynamics in a multifluid mhd model of the magnetosphere, Journal of Geophysical Research: Space Physics, 115(A10), n/a-n/a, doi: 10.1029/2010JA015579, a00J05.

Winglee, R. M. (1998), Multi-fluid simulations of the magnetosphere: The identification of the geopause and its variation with IMF, Geophys. Res. Lett., 25, 4441-4444.

Yau, A. W., T. Abe, and W. K. Peterson (2007), The polar wind: Recent observations, Journal of Atmospheric and Solar-Terrestrial Physics, 69, 1936-1983, doi: 10.1016/j.jastp.2007.08.010.

Zeng, W., and J. L. Horwitz (2007), Formula representation of auroral ionospheric o+ outflows based on systematic simulations with effects of soft electron precipitation and transverse ion heating, Geophysical Research Letters, 34(6), n/a-n/a, doi: 10.1029/2006GL028632, 106103.

Zheng, Y., T. E. Moore, F. S. Mozer, C. T. Russell, and R. J. Strangeway (2005), Polar study of ionospheric ion outflow versus energy input, Journal of Geophysical Research (Space Physics), 110, 7210-+, doi:10.1029/2004JA010995. 


\section{Regions of Outflow}

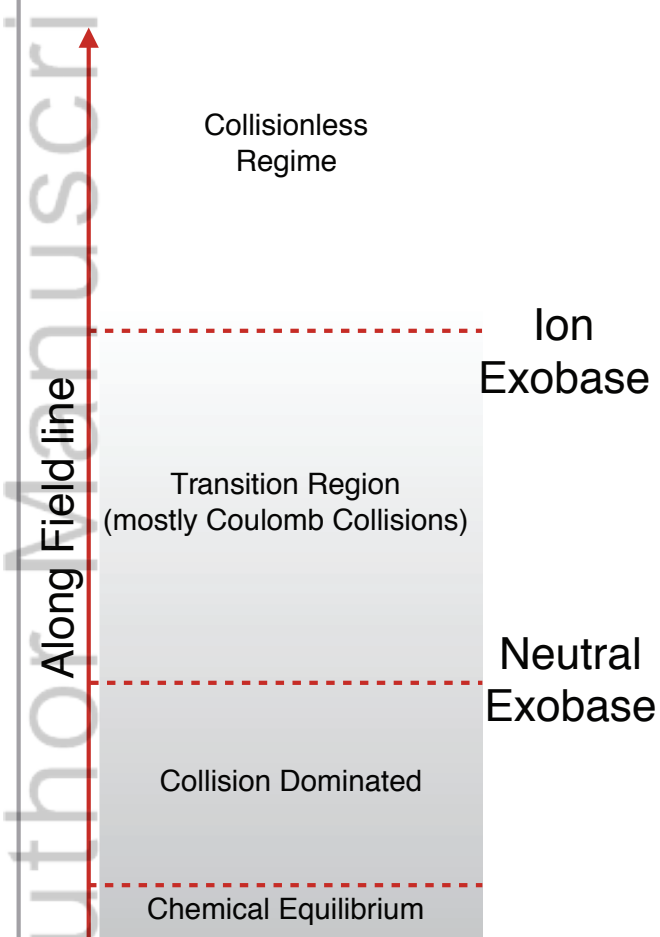

\section{Modeling Approach}

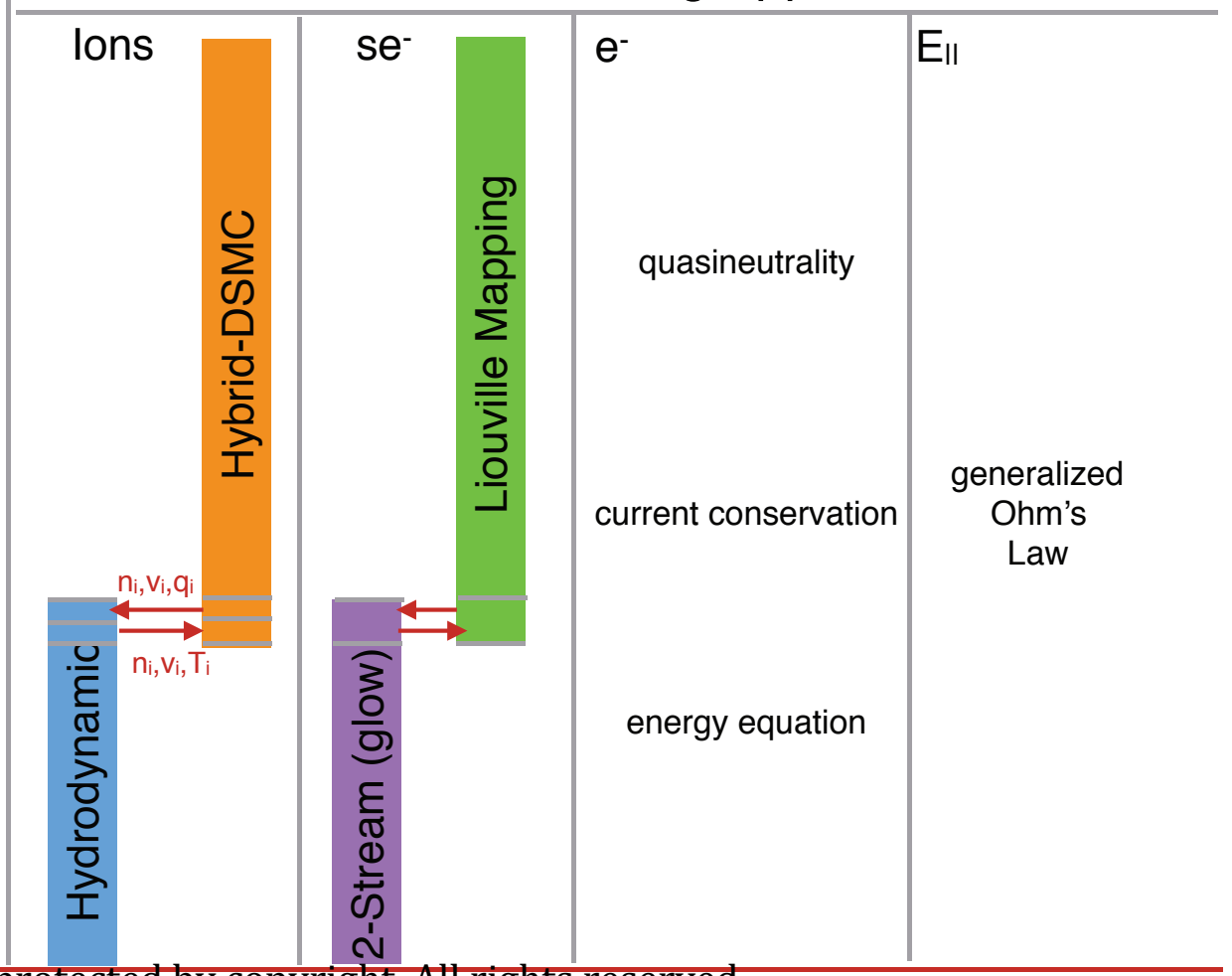

This article is protected by copyright. All rights reserved. 
Figure 2.
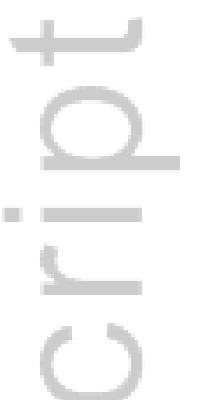

$\infty$
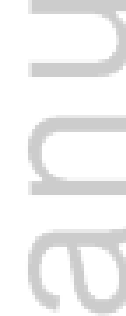

This article is protected by copyright. All rights reserved. 
Hydrodynamic

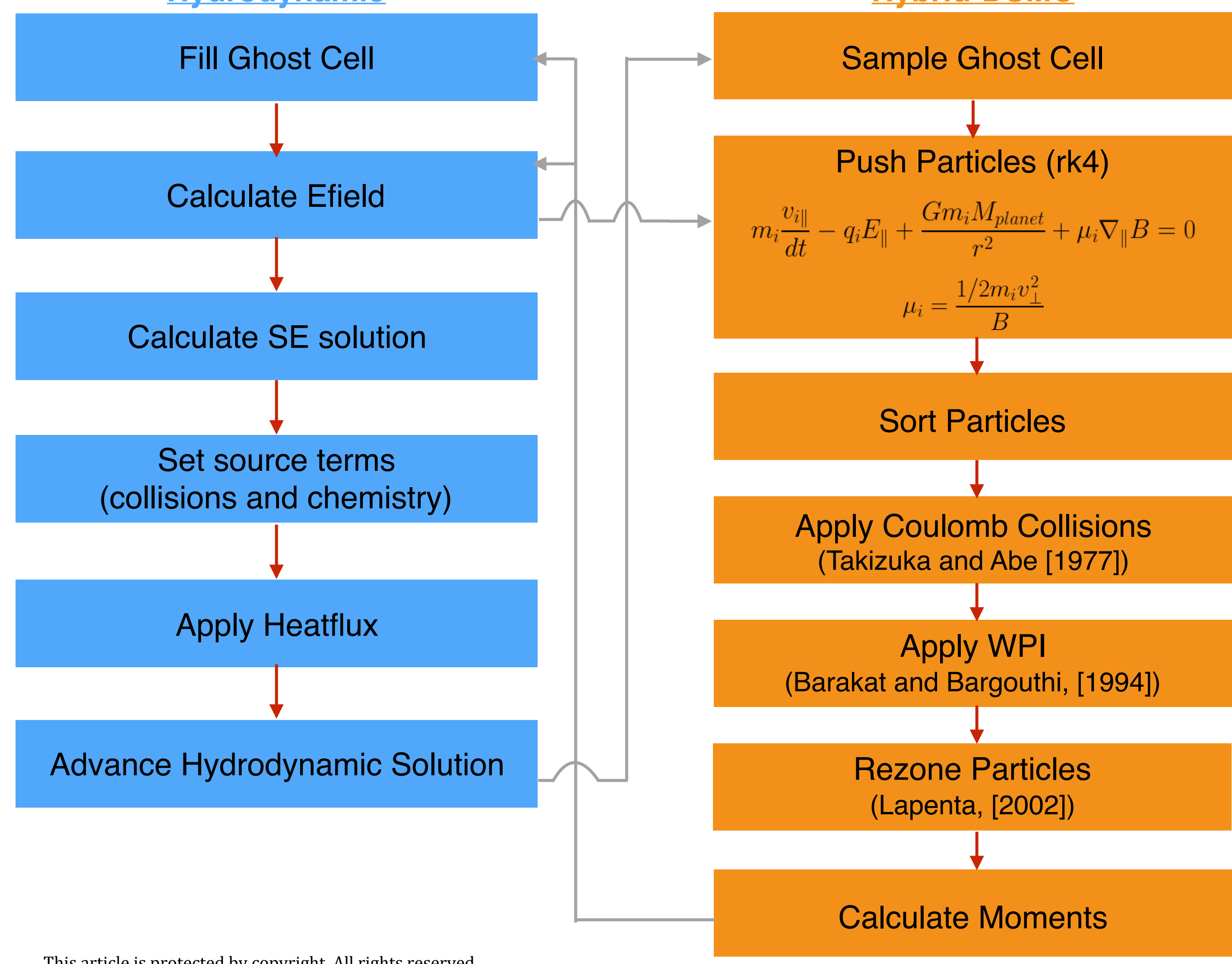




\section{OpenMP speedup of $1 \mathrm{MPI}$ proc}

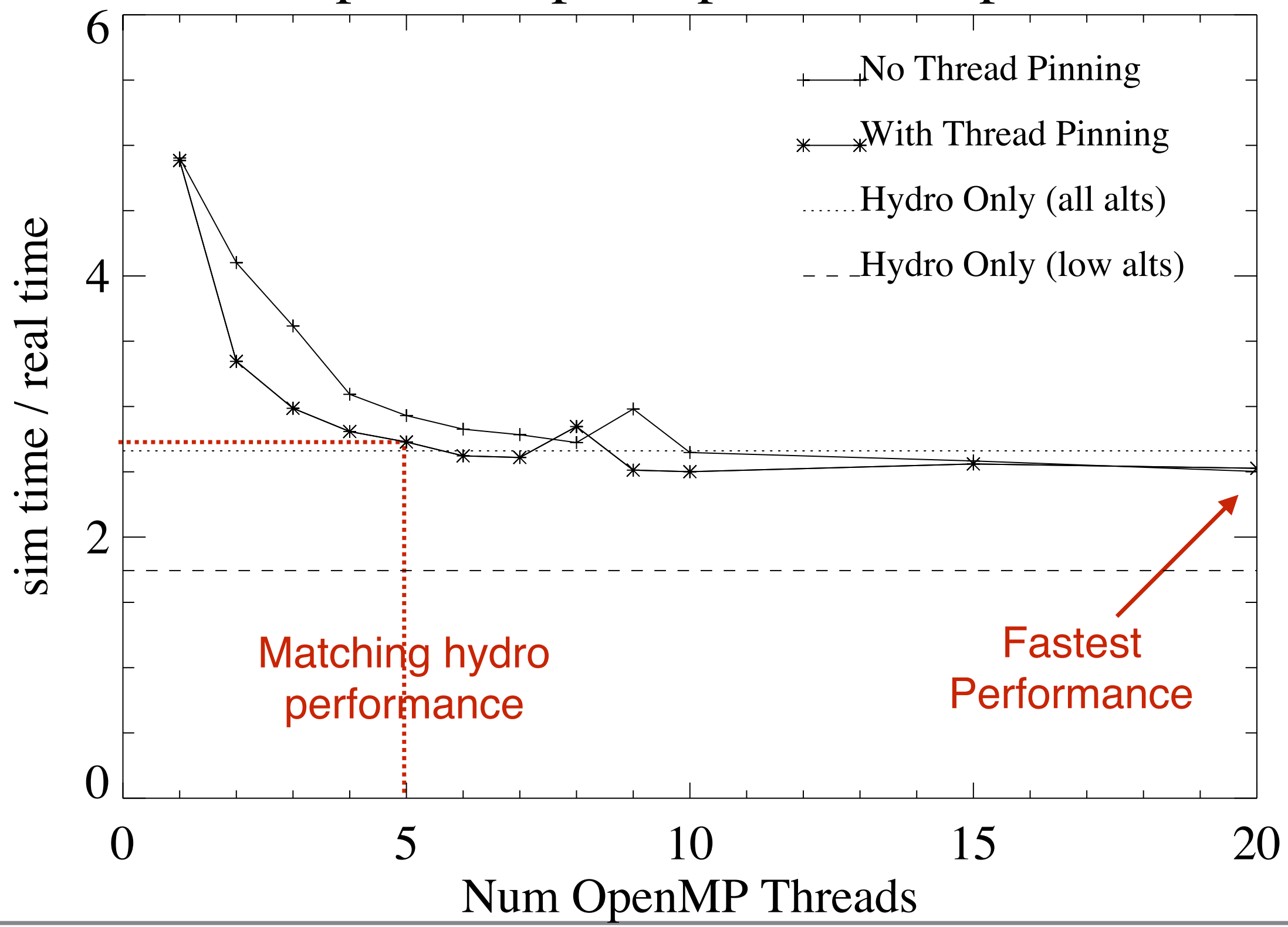


Figure 5.
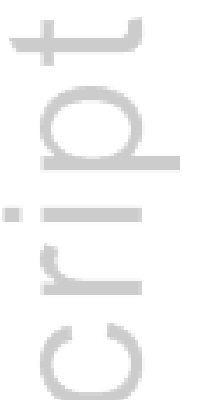

$\infty$
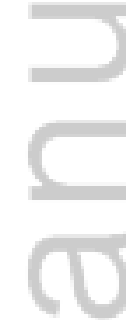

This article is protected by copyright. All rights reserved. 


\section{Comparing Kinetic and Hydrodynamic Solution}

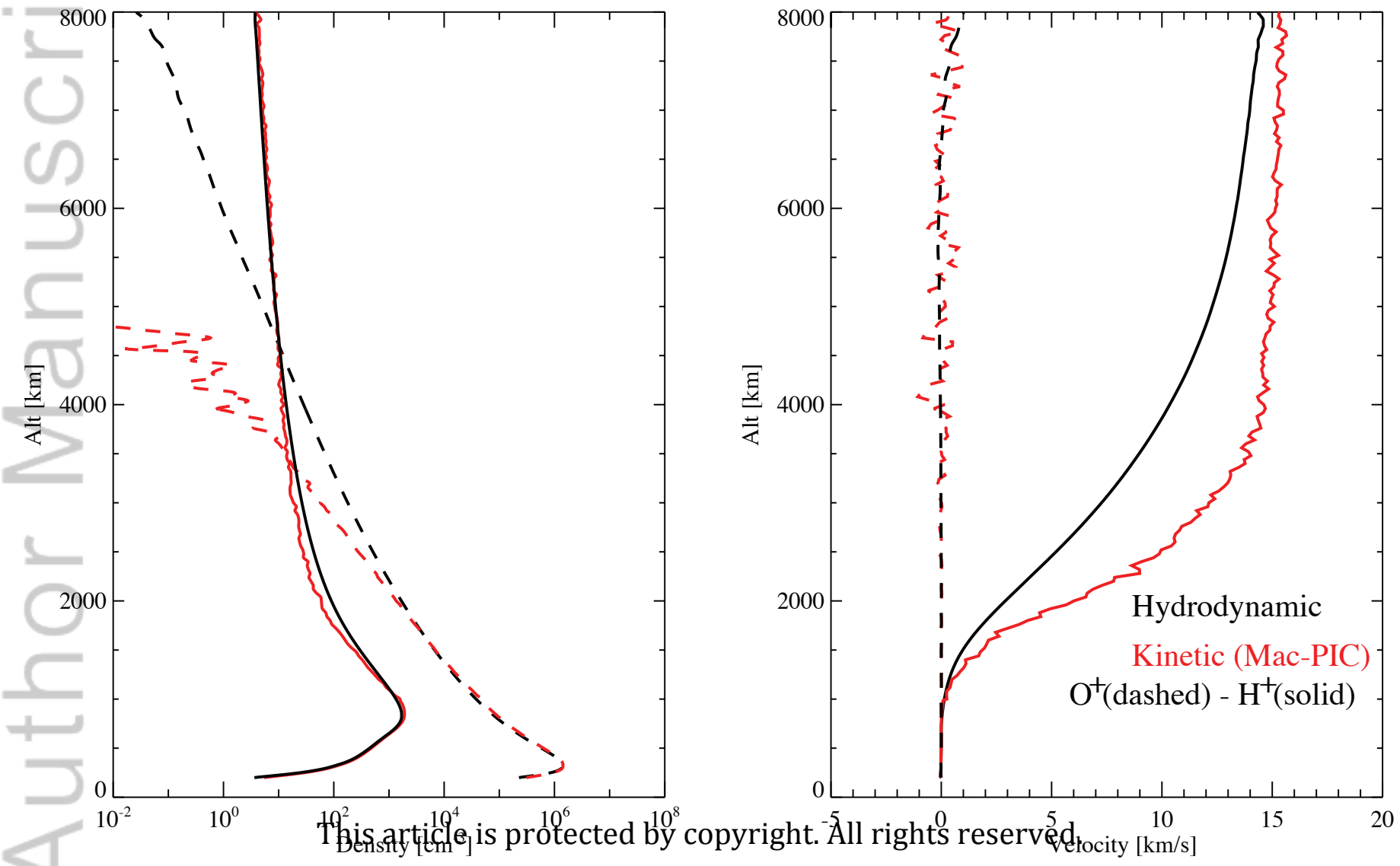




\section{Outflow Above the Cusp}

\section{Moments}
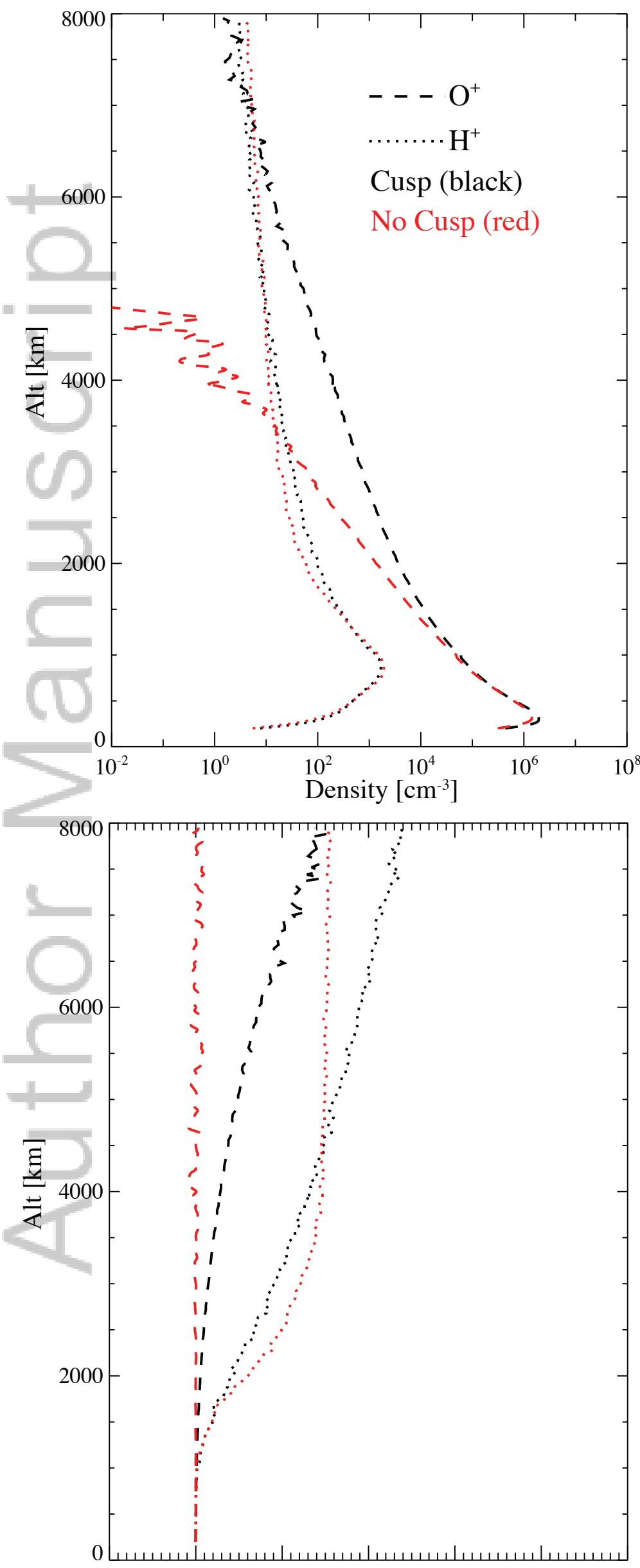

O+ Distribution Function
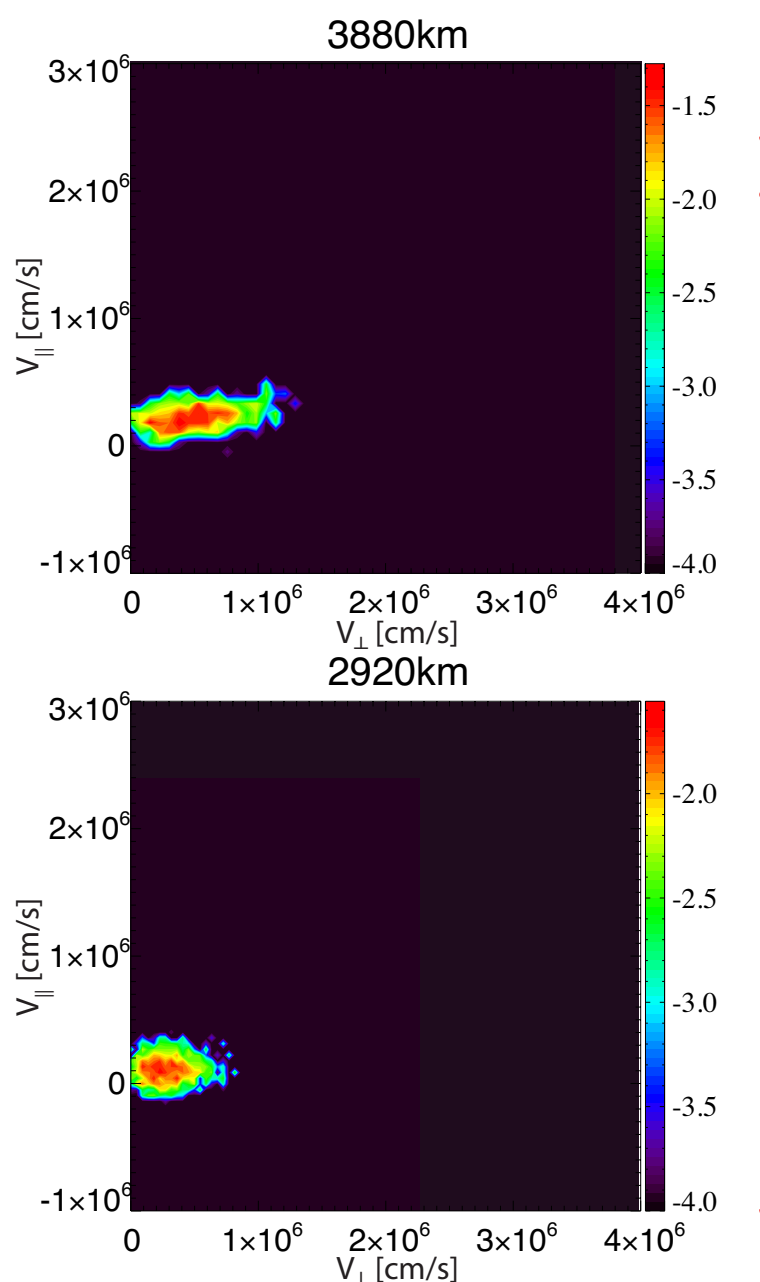
$1960 \mathrm{~km}$
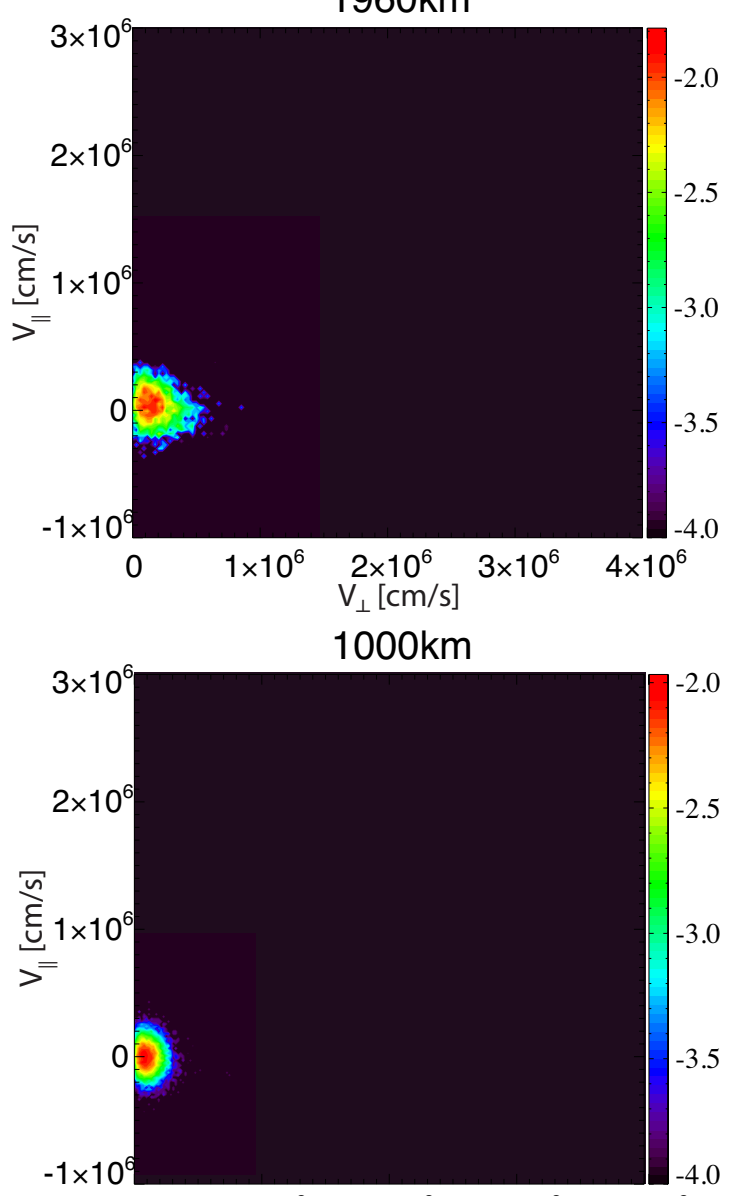


\section{Model Coupling: Particle - Fields}

Solar. Wind
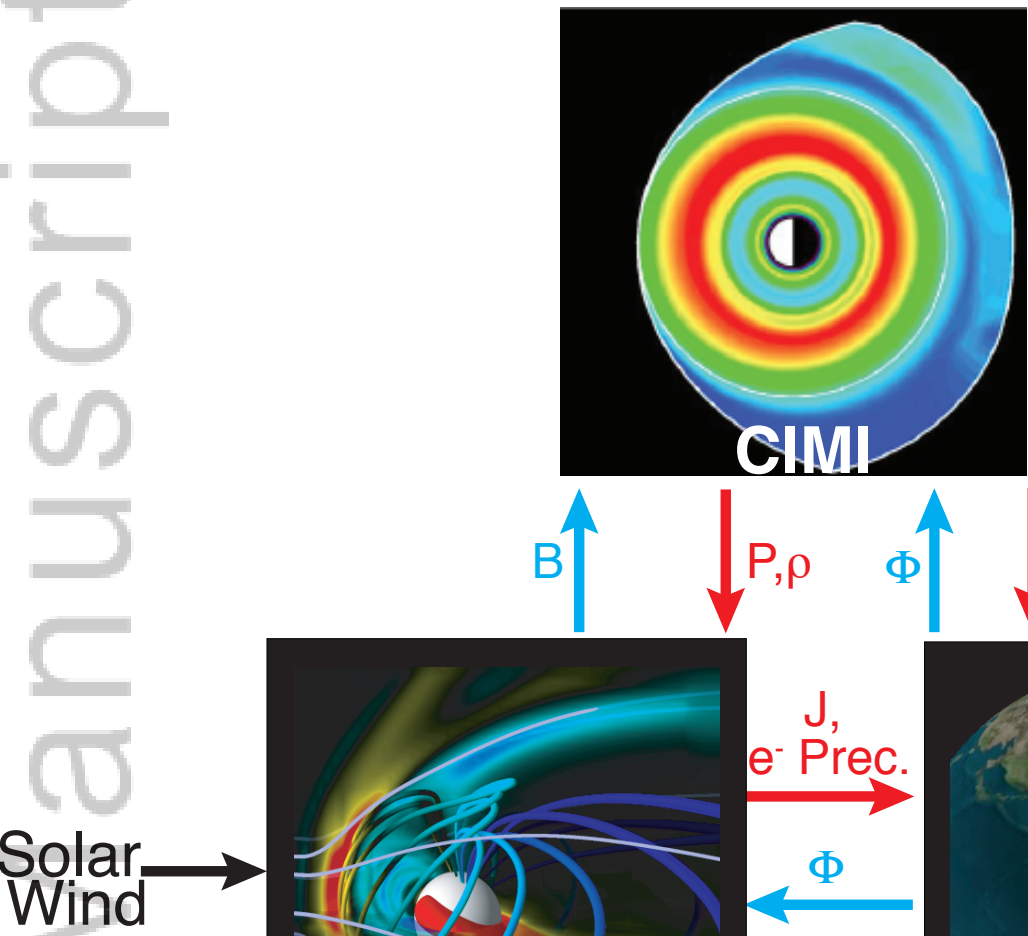

\section{BATS-R-US}
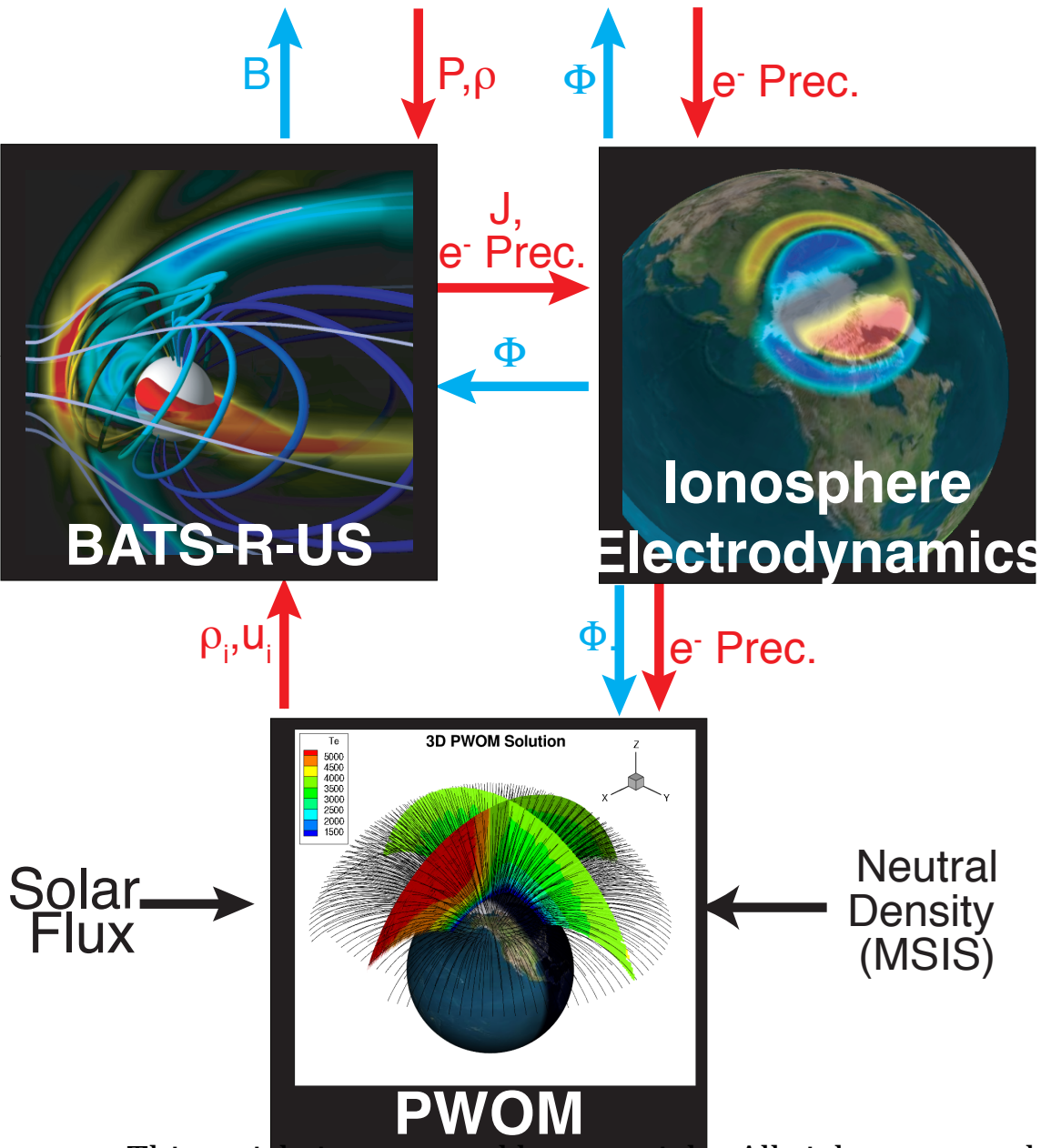

This article is protected by copyright. All rights reserved. 
Figure 9.
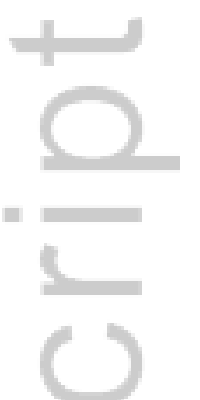

$\infty$
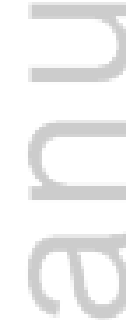

This article is protected by copyright. All rights reserved. 


\section{PWOM Output at $6000 \mathrm{~km}-$ Time $=4 \mathrm{hr}$}
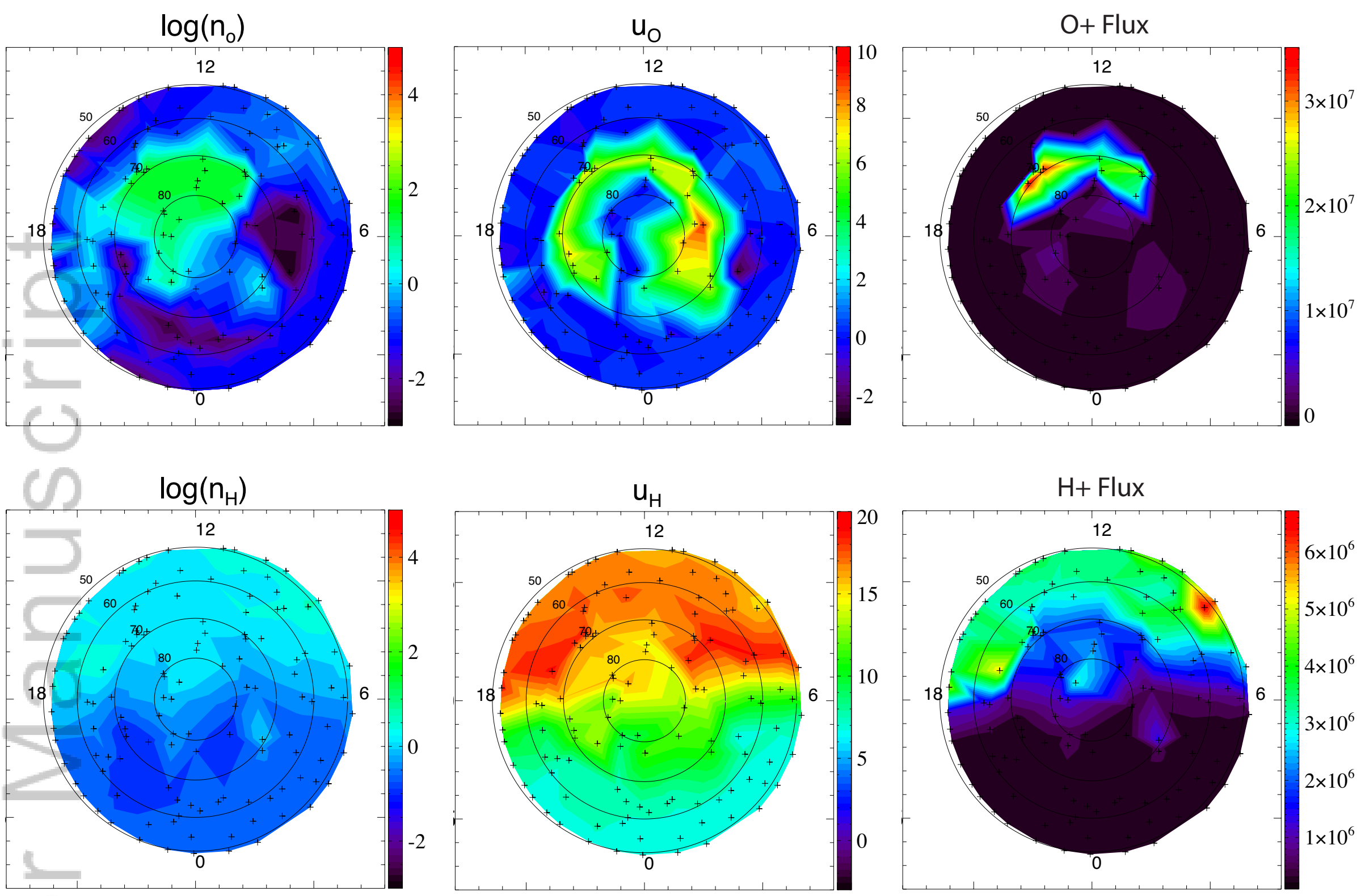

\section{Ionosphere Electrodynamics Output - Time $=4 \mathrm{hr}$}
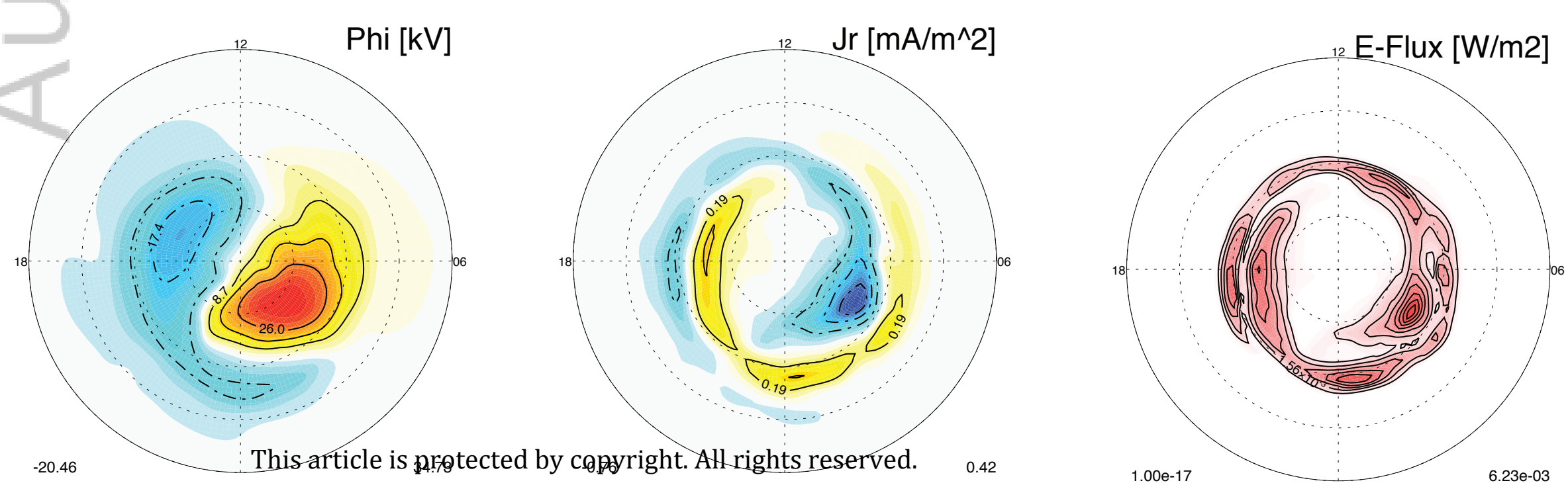


\section{Ring Current Pressure - Time $=4 \mathrm{hr}$}

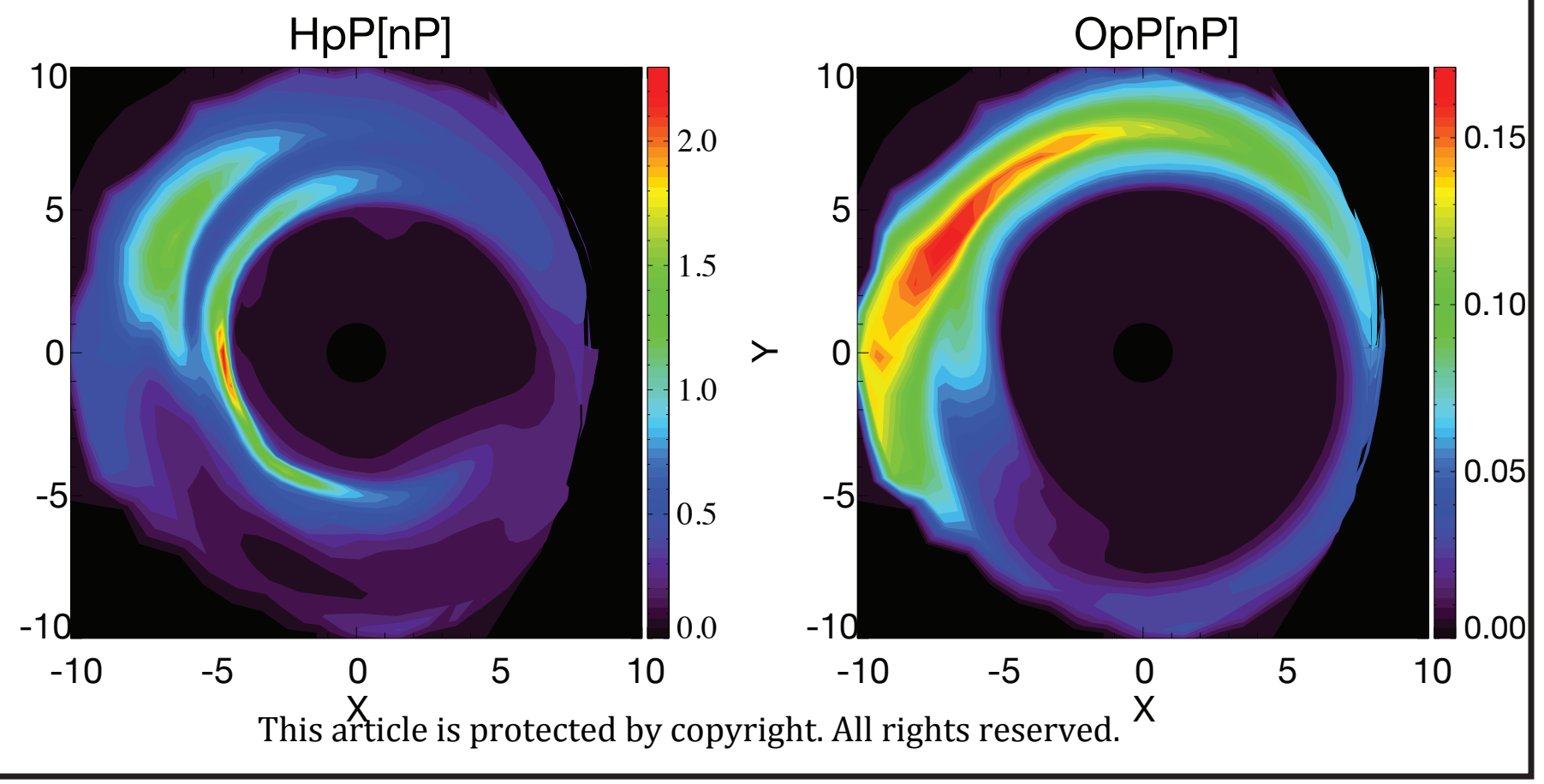

